\title{
Electric dipole moments of charged leptons with sterile fermions
}

\author{
Asmaa Abada and Takashi Toma \\ Laboratoire de Physique Théorique, CNRS, \\ Univ. Paris-Sud, Université Paris-Saclay, 91405 Orsay, France \\ E-mail: asmaa.abada@th.u-psud.fr, takashi.toma@th.u-psud.fr
}

ABSTRACT: We address the impact of sterile fermions on charged lepton electric dipole moments. Any experimental signal of these observables calls for scenarios of physics beyond the Standard Model providing new sources of CP violation. In this work, we consider a minimal extension of the Standard Model via the addition of sterile fermions which mix with active neutrinos and we derive the corresponding analytical expressions for the electric dipole moments of charged leptons at two-loop order. Our study reveals that, in order to have a non-vanishing contribution in this framework, the minimal extension necessitates the addition of at least 2 sterile fermion states to the Standard Model field content. Our conclusion is that sterile neutrinos can give significant contributions to the charged lepton electric dipole moments, some of them lying within present and future experimental sensitivity if the masses of the non-degenerate sterile states are both above the electroweak scale. The Majorana nature of neutrinos is also important in order to allow for significative contributions to the charged lepton electric dipole moments. In our analysis we impose all available experimental and observational constraints on sterile neutrinos and we further discuss the prospect of probing this scenario at low and high energy experiments.

Keywords: CP violation, Beyond Standard Model, Neutrino Physics

ARXiv EPrint: 1511.03265 


\section{Contents}

1 Introduction 1

2 The model 4

2.1 Lagrangian 4

2.2 Mixing matrix 4

3 Electric dipole moments $\quad 5$

$\begin{array}{lll}3.1 & \text { Current experimental bounds } & 6\end{array}$

$\begin{array}{lc}3.2 & \text { Calculation of charged lepton EDMs }\end{array}$

$\begin{array}{lll}3.2 .1 & \text { Diagrams } & 6\end{array}$

3.2.2 Some relevant steps in the calculation of the EDMs 9

$\begin{array}{lll}3.3 & 3+1 \text { model } & 10\end{array}$

$\begin{array}{lll}3.4 & 3+2 \text { model } & 10\end{array}$

4 Constraints 11

4.1 Lepton flavour violating processes 11

$\begin{array}{lll}4.2 & \text { Direct collider production } & 12\end{array}$

$\begin{array}{lll}4.3 & \text { Electroweak precision data } & 12\end{array}$

$\begin{array}{lll}4.4 & \text { Perturbative unitarity bound } & 13\end{array}$

$\begin{array}{ll}4.5 & \text { Other constraints } \\ \end{array}$

5 Numerical results $\quad 14$

$\begin{array}{lll}5.1 & \text { Electric dipole moments of charged leptons } & 14\end{array}$

$\begin{array}{ll}5.2 \text { Muon anomalous magnetic moment } & 17\end{array}$

$\begin{array}{lll}6 & \text { Summary } & 19\end{array}$

$\begin{array}{ll}\text { A Loop calculations } & 19\end{array}$

\section{Introduction}

After the discovery of the Higgs boson at the LHC [1, 2], the quest of new physics beyond the Standard Model (SM) is being pursued in many fronts. Indeed, other than neutrino oscillation phenomena, there are several observational problems and theoretical caveats suggesting that new physics is indeed required: the former are related to the baryon asymmetry of the Universe and the need for a dark matter candidate, while among the latter one can mention the hierarchy problem, the flavour puzzle, or fine-tuning in relation to electroweak (EW) symmetry breaking. Disentangling the new physics models and in particular, probing the underlying neutrino mass generation mechanism, requires investigating 
all available observables, arising from all avenues - high-intensity, high-energy and cosmology - as well as thoroughly exploring the interplay between them.

There are numerous well-motivated and appealing models of new physics that aim at addressing these issues, and which are currently being actively investigated and searched for. Focusing on the neutrino oscillation phenomena, in order to account for neutrino masses and mixings, many extensions of the SM call upon the introduction of right-handed neutrinos - which are sterile states - giving rise to a Dirac mass term for the neutral leptons. One of the most economical possibility is the embedding of the (standard or type I) seesaw mechanism [3-9] into the SM. These scenarios cannot be probed directly: in order to have natural Yukawa couplings for the neutral leptons, the mass of the right-handed neutrinos must be in general very high, close to the gauge coupling unification scale, thus implying that direct experimental tests of the seesaw hypothesis are virtually impossible. In contrast, low-scale seesaw models [10-16], in which right-handed or sterile fermion states with masses around the electroweak scale or even much lower are added to the SM, prove to be very attractive since the new states can be produced in colliders and/or in low-energy experiments, and their contributions to physical processes can be sizable, provided that a non-negligible mixing to the (mostly) active neutrinos is present. This is the case for the $\nu$ MSM [10], the Inverse Seesaw [11], the Linear Seesaw [17, 18] and the low-scale type-I seesaw $[14,15]$.

Some of the latter models may also explain the relic density of dark matter taking the lightest keV scale sterile neutrino as a candidate [10, 19] and the baryon asymmetry of the Universe obtained via leptogenesis through neutrino oscillations and CP violating phases in the lepton sector [20-23]. In addition, low scale ( $\mathrm{GeV}$-scale) leptogenesis with 3 sterile neutrinos has also been discussed [24].

Present in several neutrino mass models, the sterile fermion masses can range from well below the electroweak scale $(\mathrm{GeV})$ up to the Planck scale. Their existence is strongly motivated by current reactor [25-27], accelerator [28-31] and Gallium anomalies [32, 33] suggesting that there might be some extra fermionic gauge singlets with mass(es) in the $\mathrm{eV}$ range. Other than the reactor and accelerator anomalies, their existence is also motivated by indications from large scale structure formation [34, 35]. Moreover and depending on their masses, sterile fermion states present in these extensions can also give rise to interesting collider signatures [36-48]. Nevertheless, due to the mixings of the sterile states with the active left-handed neutrinos, models with sterile fermions are severely constrained from electroweak precision observables, laboratory data and cosmology.

These extensions of the SM with sterile fermions aiming at incorporating massive neutrinos and the leptonic mixing may also open the door to many new phenomena, such as flavour violation in the charged lepton sector and contributions to lepton flavour-conserving observables such as charged lepton electric and magnetic dipole moments.

In this work, we study the effect of sterile fermions which mix with active neutrinos on the electric dipole moments (EDMs) of charged leptons. An EDM, which corresponds to the coupling of the spin of a particle to an external electric field, is a flavour conserving observable which may provide a clear signal of the existence of extra $\mathrm{CP}$ violating phases. 
Exploring the origin of $\mathrm{CP}$ violation is important in extending the SM. The CP violating observables which have been identified in Kaon and B meson systems are consistent with the SM explanations and their measured values can be accommodated with the unique source of $\mathrm{CP}$ violation of the SM. ${ }^{1}$ The amount of $\mathrm{CP}$ violation in the SM can be parametrised by the Jarlskog invariant $J_{\mathrm{CP}}$. However, in order to explain the observed baryon asymmetry of the Universe, additional CP-violating sources are required. Extensions of the SM accommodating neutrino masses and mixings might provide additional sources of $\mathrm{CP}$ violation. In fact, by itself, the parametrisation of the leptonic mixing matrix in terms of 3 mixing angles and one Dirac CP violating phase (plus two additional ones in the case where the neutrinos are of Majorana nature) already opens the possibility of $\mathrm{CP}$ violation in the leptonic sector.

For this study, we consider a minimal extension of the SM via the addition of $N$ of sterile fermions which mix with the active neutrinos, and we address their impact on the charged lepton EDMs. In our chosen framework, we do not impose any seesaw realization, meaning no hypothesis is made on the underlying mechanism of neutrino masses and mixings generation, only assuming that the physical and the interaction neutrino basis are related via a $(3+N) \times(3+N)$ unitary mixing matrix, which reduces to the PontcorvoMaki-Nakagawa-Sakata (PMNS) matrix, $U_{\mathrm{PMNS}}$, in the case of three neutrino generations (no additional sterile neutrinos are present). Since we consider that neutrino mass eigenvalues and the lepton mixing matrix are independent, this simple model can be seen as an "effective" model allowing to investigate the potential imprints (on EDMs) due to the presence of a number $N$ of sterile neutrinos present in several new physics scenario including Type-I seesaw, inverse seesaw and linear seesaw models. The computation of EDMs in the presence of right-handed neutrinos has been extensively discussed in refs. [49-51], and that in the context of supersymmetric seesaw has been discussed in refs. [52-54].

In the presence of massive sterile neutrinos, charged lepton EDMs are induced at twoloop level. We have computed the corresponding diagrams - providing the corresponding analytical expressions for the EDMs of charged leptons at two-loop order - and we have shown that in order to have a significant contribution, the minimal extension of the SM indeed requires the addition of at least 2 sterile fermion states. We have also shown that the Majorana nature of neutrinos is also important in order to allow for significative contribution to the charged lepton EDMs. We complete our analysis by also discussing the several experimental and theoretical constraints on our scenario including those from charged lepton flavour violating (cLFV) processes, direct collider searches, electroweak precision data and the perturbative unitarity constraint. We confront our findings to the current experimental status and we conclude that, depending on their masses and on the active-sterile mixing angles, sterile neutrinos can give significant contributions to the charged lepton EDMs, some of these observables even lying within present and future experimental sensitivity.

\footnotetext{
${ }^{1}$ There is an additional CP violating term $\mathcal{L}=\theta_{\mathrm{QCD}} \frac{\alpha_{s}}{8 \pi} G_{\mu \nu}^{a} \tilde{G}^{a \mu \nu}$, where $\tilde{G}^{a \mu \nu} \equiv \frac{1}{2} \epsilon^{\mu \nu \rho \sigma} G_{\rho \sigma}^{a}$ is the dual tensor of the field strength tensor of gluon $G_{\mu \nu}^{a}$ and $\alpha_{s}$ is the strong coupling constant. This coupling $\left(\theta_{\mathrm{QCD}}\right)$ should be $\theta_{\mathrm{QCD}} \lesssim 10^{-10}$ due to the constraint of the neutron EDM.
} 


\section{The model}

In order to accommodate neutrino masses and mixings, the SM can be extended with new sterile fermions such as right-handed (Majorana) neutrinos. In this work, we consider the SM extended by $N$ sterile fermion states which mix with the three active neutrinos. We consider that the neutrino mass eigenvalues and the lepton mixing matrix are independent, meaning that no assumption is made on the neutrino mass generation mechanism. ${ }^{2}$ As we will see later, we focus on the $3+1(N=1)$ and $3+2(N=2)$ models. For $N>2$, we expect that the results do not change with respect to the $N=2$ case.

\subsection{Lagrangian}

After electroweak symmetry breaking, the relevant terms in the Lagrangian can be written in the Feynman-'t Hooft gauge as

$$
\begin{aligned}
\mathcal{L}= & -\frac{g_{2}}{\sqrt{2}} U_{\alpha i} W_{\mu}^{-\overline{\ell_{\alpha}}} \gamma^{\mu} P_{L} \nu_{i}-\frac{g_{2}}{\sqrt{2}} U_{\alpha i} H^{-} \overline{\ell_{\alpha}}\left(\frac{m_{\alpha}}{m_{W}} P_{L}-\frac{m_{i}}{m_{W}} P_{R}\right) \nu_{i}+\text { H.c. } \\
& -\frac{g_{2}}{2 \cos \theta_{W}} U_{\alpha i}^{*} U_{\alpha j} Z_{\mu} \overline{\nu_{i}} \gamma^{\mu} P_{L} \nu_{j}-\frac{i g_{2}}{2} U_{\alpha i}^{*} U_{\alpha j} A^{0} \overline{\nu_{i}}\left(\frac{m_{j}}{m_{W}} P_{R}\right) \nu_{j}+\text { H.c. } \\
& -\frac{g_{2}}{2} U_{\alpha i}^{*} U_{\alpha j} h \overline{\nu_{i}}\left(\frac{m_{j}}{m_{W}} P_{R}\right) \nu_{j}+\text { H.c. }
\end{aligned}
$$

where $g_{2}$ is the $\mathrm{SU}(2)_{L}$ gauge coupling, $U_{\alpha i}$ is the $3 \times(3+N)$ lepton mixing matrix, $m_{i}$ is the mass eigenvalue of the neutrinos and $m_{\alpha}$ is the charged lepton mass. The indices $\alpha$ and $i, j$ run $\alpha=e, \mu, \tau$ and $i, j=1, \cdots, 3+N$. The mixing matrix $U_{\alpha i}$ obeys the following relations due to unitarity conditions:

$$
\sum_{i=1}^{3+N} U_{\alpha i} U_{\beta i}^{*}=\delta_{\alpha \beta}, \quad \sum_{\alpha=e, \mu, \tau} U_{\alpha i}^{*} U_{\alpha j} \neq \delta_{i j},
$$

Further details can be found in, for example, refs. [55, 56]. Although the above Lagrangian in eq. (2.1) has been derived by assuming Type-I seesaw mechanism in ref. [56], it is also valid for Inverse seesaw and Linear seesaw mechanisms as well. The difference among these mechanisms is reflected in the mixing matrix $U_{\alpha i}$ obtained after the diagonalisation of the corresponding neutrino mass matrix. However eq. (2.1) is not valid for a (pure) Type-II seesaw mechanism due to the presence of $\mathrm{SU}(2)_{L}$ scalar triplet(s) instead of sterile fermions.

\section{$2.2 \quad$ Mixing matrix}

In the $3+N$ model, the mixing matrix $U$ includes $(3+N)(2+N) / 2$ rotation angles, $(2+N)(1+N) / 2$ Dirac phases and $2+N$ Majorana phases. As an example, the mixing matrix $U$ for the $N=2$ can be parametrised as

$$
U=R_{45} R_{35} R_{25} R_{15} R_{34} R_{24} R_{14} R_{23} R_{13} R_{12} \operatorname{diag}\left(1, e^{i \varphi_{2}}, e^{i \varphi_{3}}, e^{i \varphi_{4}}, e^{i \varphi_{5}}\right),
$$

\footnotetext{
${ }^{2}$ Should we have considered a given neutrino mass generation mechanism, the physical neutrino masses and the lepton mixing matrix $U$ would be derived from the diagonalisation of the full $(3+N) \times(3+N)$ neutrino mass matrix and thus be related - provided one respects neutrino data in what concerns the active (light) neutrinos.
} 
where $R_{i j}$ is the rotation matrix between $i$ and $j$. For instance, the rotation matrix $R_{45}$ is explicitly given by

$$
R_{45}=\left(\begin{array}{ccccc}
1 & 0 & 0 & 0 & 0 \\
0 & 1 & 0 & 0 & 0 \\
0 & 0 & 1 & 0 & 0 \\
0 & 0 & 0 & \cos \theta_{45} & \sin \theta_{45} e^{-i \delta_{45}} \\
0 & 0 & 0 & -\sin \theta_{45} e^{i \delta_{45}} & \cos \theta_{45}
\end{array}\right),
$$

and likewise for the other matrices $R_{i j}$ (in terms of $\theta_{i j}$ and $\delta_{i j}$ ).

Since the number of Dirac phases is 6 for the case where $N=2$, four Dirac phases $\delta_{i j}$ can be eliminated. In this paper, we set $\delta_{12}=\delta_{23}=\delta_{24}=\delta_{45}=0$. The mixing matrix for $N=1$ can be obtained by taking the $4 \times 4$ sub-matrix after substituting $R_{i 5}=\mathbb{1}$ in eq. (2.3).

According to a global analysis of solar, atmospheric, reactor and accelerator neutrino data, the best fit values of the mixing angles and neutrino mass differences for normal hierarchy are given by [57],

$$
\begin{aligned}
\sin ^{2} \theta_{12} & =0.304, \quad \sin ^{2} \theta_{23}=0.452, \quad \sin ^{2} \theta_{13}=0.0218, \\
\Delta m_{21}^{2} & =7.50 \times 10^{-5} \mathrm{eV}, \quad \Delta m_{31}^{2}=2.457 \times 10^{-3} \mathrm{eV},
\end{aligned}
$$

and for inverted hierarchy are given by

$$
\begin{aligned}
\sin ^{2} \theta_{12} & =0.304, \quad \sin ^{2} \theta_{23}=0.579, \quad \sin ^{2} \theta_{13}=0.0219, \\
\Delta m_{21}^{2} & =7.50 \times 10^{-5} \mathrm{eV}, \quad \Delta m_{31}^{2}=-2.449 \times 10^{-3} \mathrm{eV} .
\end{aligned}
$$

We use these values for our numerical analyses. Note that the following EDM computation does not substantially depend on neutrino mass hierarchies since, as we will see, the heavy sterile neutrinos give the dominant contributions to the EDMs.

In our work we have varied all the phases ${ }^{3}$ including $\delta_{13}$ in the range $[0,2 \pi]$.

\section{Electric dipole moments}

A non-zero value of the EDM for elementary particles implies violations of parity $(\mathrm{P})$ and time reversal $(\mathrm{T})$ symmetries. This is translated to $\mathrm{CP}$ violation due to requirement of CPT invariance. In the SM, the electron EDM is induced at four-loop level through the Jarlskog invariant $J_{\mathrm{CP}}$, and the predicted value is approximately given by $[58,59]$

$$
\left|d_{e}\right| / e \sim \frac{\alpha_{W}^{3} \alpha_{s} m_{e}}{256(4 \pi)^{4} m_{W}^{2}} J_{\mathrm{CP}} \sim 3 \times 10^{-38} \mathrm{~cm}
$$

where the Jarlskog invariant is defined with the CKM matrix $V$ by

$$
J_{\mathrm{CP}} \equiv\left|\operatorname{Im}\left(V_{u s} V_{c s}^{*} V_{c b} V_{u b}^{*}\right)\right|=\sin \theta_{12}^{q} \sin \theta_{23}^{q} \sin \theta_{13}^{q} \cos \theta_{12}^{q} \cos \theta_{23}^{q} \cos ^{2} \theta_{13}^{q} \sin \delta_{q},
$$

with the rotation angles $\theta_{12}^{q}, \theta_{23}^{q}, \theta_{13}^{q}$ and the Dirac CP phase $\delta_{q}$ in the quark sector. The value of the Jarlskog invariant obtained from the global fit of the CKM matrix elements is $J_{\mathrm{CP}}=3 \times 10^{-5}$ [60]. The electron EDM value in eq. (3.1) is too small compared to the current experimental limit $\left|d_{e}\right| / e<8.7 \times 10^{-29} \mathrm{~cm}$ set by ACME Collaboration [61].

\footnotetext{
${ }^{3}$ The fitting of the $\mathrm{CP}$ violating Dirac phase $\delta_{13}$ also has been done in ref. [57], and the Dirac phase is in the range of $236^{\circ}<\delta_{13}<345^{\circ}$ at $1 \sigma$ level, but all values are allowed at $3 \sigma$ level.
} 


\subsection{Current experimental bounds}

The present experimental upper bounds for the charged lepton EDMs are given by

$$
\begin{aligned}
\left|d_{e}\right| / e<8.7 \times 10^{-29}[\mathrm{~cm}] & (\text { ACME) }, \\
\left|d_{\mu}\right| / e<1.9 \times 10^{-19}[\mathrm{~cm}] & \text { (Muon } g-2), \\
\left|\operatorname{Re}\left(d_{\tau}\right)\right| / e<4.5 \times 10^{-17}[\mathrm{~cm}] & \text { (Belle), } \\
\left|\operatorname{Im}\left(d_{\tau}\right)\right| / e<2.5 \times 10^{-17}[\mathrm{~cm}] & \text { (Belle). }
\end{aligned}
$$

The bounds for the electron, the muon and the tau EDMs have been measured by the ACME Collaboration [61], the Muon $(g-2)$ Collaboration [62] and the Belle Collaboration $[60,63]$ respectively. The bound for the electron EDM is especially strong. EDMs are normally understood as real numbers since one works under the assumption of CPT invariance. However, the Belle Collaboration is attempting to measure an effect of CPT violation as well, which implies violation of Lorentz invariance [64] and thus, they also give an upper bound on $\operatorname{Im}\left(d_{\tau}\right)$ as a CPT violating parameter.

The next generation ACME experiment is expected to be able to reach a sensitivity for the electron $\mathrm{EDM} \sim\left|d_{e}\right| / e \lesssim \mathcal{O}\left(10^{-30}\right) \mathrm{cm}$ with more molecules and smaller systematics [65]. The future sensitivity for the muon EDM is $\left|d_{\mu}\right| / e \sim 10^{-21} \mathrm{~cm}$ by J-PARC $g-2 /$ EDM Collaboration [66].

\subsection{Calculation of charged lepton EDMs}

In the $3+N$ model, EDMs for the charged leptons are not induced at one-loop level since the relevant amplitude is always proportional to $\left|U_{\alpha i}\right|^{2}$ which is a purely real number. Imaginary parts of the amplitude are essential for EDMs. Thus, the leading contributions to charged lepton EDMs are given at two-loop level and the relevant diagrams are depicted in figure 1. In this work, we compute all the latter contributions in the Feynman-'t Hooft gauge meaning that each of the diagrams of figure 1 stands for all possible combinations arising when one (or more) gauge boson is replaced by its corresponding Goldstone boson.

\subsubsection{Diagrams}

Some comments are in order before proceeding to the computation of the EDMs:

- The diagrams (a1), (a2), (a3), (b1) and (b2) exist only if the neutrinos are Majorana fermions, and give a non-zero EDM. On the other hand diagrams (c1) and (c2) are non-zero for both cases of Majorana and Dirac neutrinos. If, in analogy to what occurs in the quark sector, the model includes a single Dirac CP-violating phase in the lepton sector, the contribution of the diagrams (c1) and (c2) would be zero as discussed in detail in ref. [67].

- In our computation, the diagrams (d1), (d2), (d3), (d4), (e1) (e2), (e3) and (e4), mediated by the $Z$ and the Higgs bosons (last two lines in figure 1) will give similar (same order) contributions compared to the ones of the diagrams (a1), (a2), (a3), (b1), (b2), (c1) and (c2) - the only difference being a charged lepton propagator for 


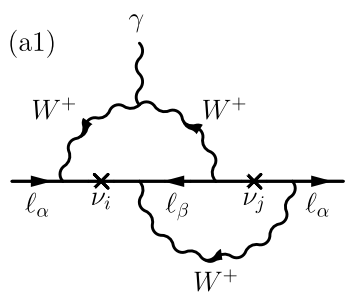

(a2)

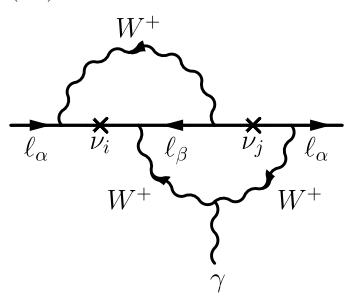

(b1)

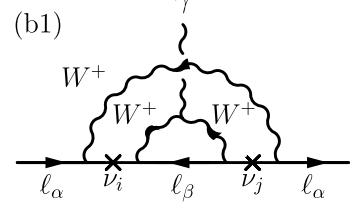

(b2)

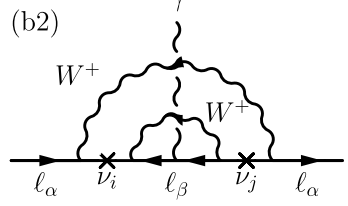

(c1)

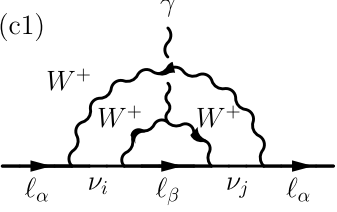

$(\mathrm{c} 2)$

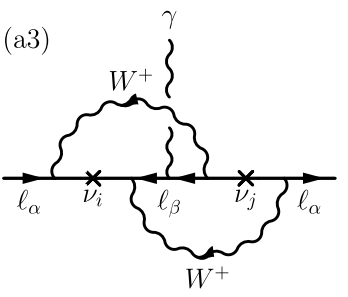

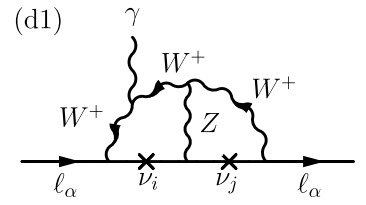
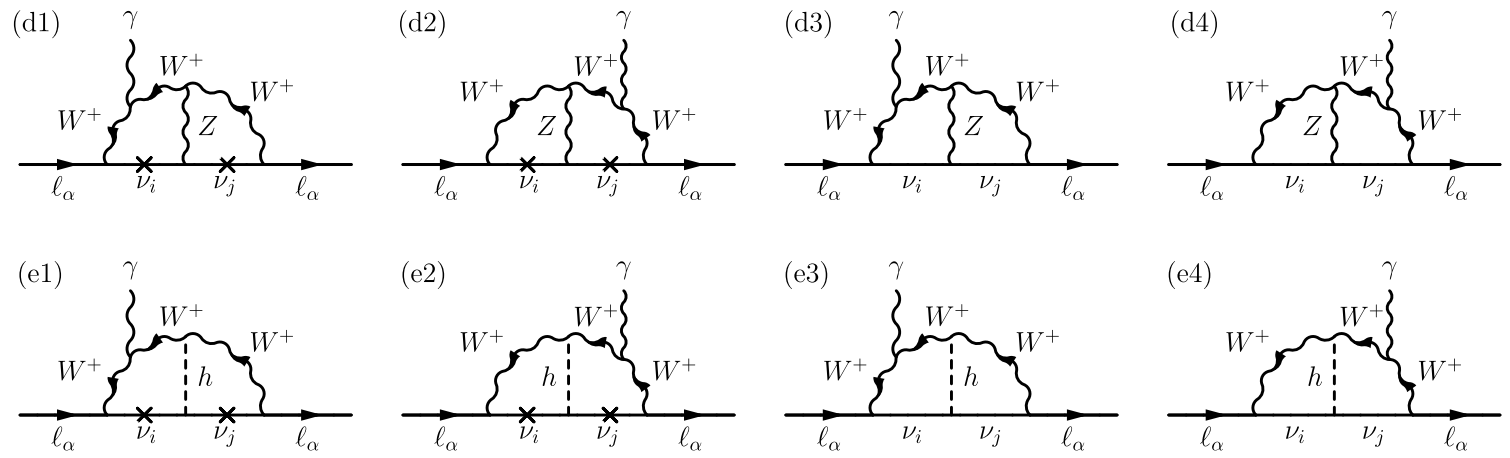

Figure 1. Diagrams contributing to charged lepton EDMs at two-loop level. The cross in the $\nu_{i}$, $\nu_{j}$ propagators means lepton number violation due to Majorana neutrino nature.

the latter diagrams and the extra Higgs or $Z$ boson propagators for the former ones. Should one include them in the computation, their effect would translate into a small factor difference at most and will not change qualitatively the results. In addition, for diagrams (e) (the last line of diagrams of figure 1), with the Higgs propagating in the loops, the Goldstone modes give rise to contributions of the order of the ones from diagrams (a), (b) and (c), as well as an additional contribution due to the Higgs selfcoupling $h\left|H^{+}\right|^{2}$. The same argument holds for the diagrams with a $Z$ propagating in the loops of diagrams in the fourth line of figure 1, corresponding to diagrams (d), since the Weinberg angle is introduced in the corresponding contribution which also avoids systematic cancellation.

- On the other hand, the diagrams in figure 2 do not contribute to charged lepton EDMs. This can be understood as follows. The diagrams (f1), (f2) and (f3) in figure 2 include the self-energy correction of charged leptons at one-loop level. The self-energy 


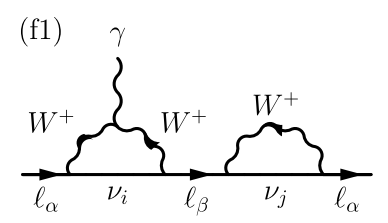

(f2)
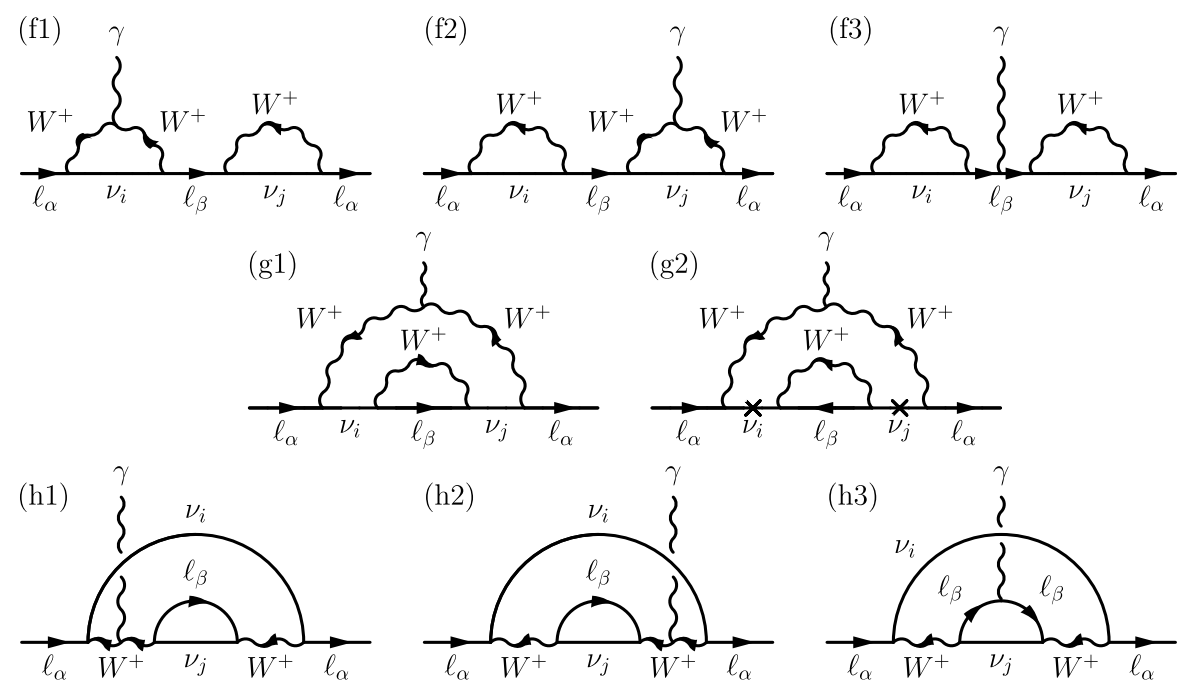

Figure 2. Diagrams not contributing to charged lepton EDMs at two-loop level.

is decomposed into two parts, a dispersive and an absorptive one. The resultant dispersive part corresponds to a renormalisation of the wave functions and of the charged lepton masses, whereas the absorptive part gives a contribution to physical quantities and exists only if the intermediate particles in the loop are on-shell. ${ }^{4}$ Since the charged leptons cannot decay into a gauge boson and the neutrino, in our case we do not have absorptive parts of the self-energy. Thus after renormalisation, the self-energy contribution becomes diagonal in terms of $\alpha$ and $\beta$, and the diagrams do not give a contribution to the EDMs since an off-diagonal component $(\alpha \neq \beta)$ is needed in order to have a contribution.

- For the diagrams (g1) and (g2) in figure 2, the relevant amplitudes for EDMs are in general factorised into CP phase factors and dimensionless loop functions after some calculations. As we will see later, the CP phase factors and loop functions should be both anti-symmetric under the exchange $i \leftrightarrow j$ in order to give a non-zero contribution to the EDM ( $i, j$ referring to the neutrino mass eigenstates). However, since the resulting loop functions for the diagrams (g1) and (g2) are fully symmetric under the exchange $i \leftrightarrow j$, their contributions to EDMs are thus zero.

- The diagrams (h1), (h2) and (h3) in figure 2 do not contribute to EDMs since, as one can notice, their amplitudes are always proportional to $\left|U_{\alpha i}\right|^{2}\left|U_{\beta j}\right|^{2}$, which are real quantities.

As a consequence of the above discussion, we are left with 44 two-loop diagrams for (a1), (a2), (a3), (b1), (b2), (c1) and (c2), taking into account longitudinal modes of the Goldstone boson. (Should we take into account the $Z$ and Higgs bosons contributions, which correspond to the diagrams (d1), (d2), (d3), (d4), (e1) (e2), (e3) and (e4) of figure 1, this

\footnotetext{
${ }^{4}$ Leptogenesis is one of the examples for such a case $[68,69]$.
} 
would correspond to 96 two-loop diagrams. However, as mentioned above, we do not consider the latter diagrams (d) and (e).) While some additional diagrams given by four-point vertices $h-H^{ \pm}-W^{\mp}-\gamma, Z-H^{ \pm}-H^{\mp}-\gamma, A^{0}-H^{ \pm}-W^{\mp}-\gamma Z-W^{ \pm}-W^{\mp}-\gamma$ also exist, we have checked that these contributions are negligible compared to the relevant diagrams we considered.

\subsubsection{Some relevant steps in the calculation of the EDMs}

We perform the computation of the charged lepton EDMs as follows. After having written the loop integrals with the help of the Feynman parameters, the simplification of the amplitude is done with FeynCalc [70]. Finally, one can extract the relevant terms to the EDMs,

$$
i \mathcal{M}=d_{\alpha} \epsilon_{\mu}^{*}(q) \bar{u}\left(p_{2}\right) i \sigma^{\mu \nu} q_{\nu} \gamma_{5} u\left(p_{1}\right)
$$

where $d_{\alpha}$ is the EDM of a charged lepton $\ell_{\alpha}, p_{1}$ and $p_{2}$ are the initial and final momentum of the charged lepton, respectively, and finally, $q\left(q=p_{2}-p_{1}\right)$ is the photon momentum.

In the $3+N$ model, the EDM of a charged lepton $\ell_{\alpha}$ can be expressed by

$$
d_{\alpha}=-\frac{g_{2}^{4} e m_{\alpha}}{4(4 \pi)^{4} m_{W}^{2}} \sum_{\beta} \sum_{i, j}\left[J_{i j \alpha \beta}^{M} I_{M}\left(x_{i}, x_{j}, x_{\alpha}, x_{\beta}\right)+J_{i j \alpha \beta}^{D} I_{D}\left(x_{i}, x_{j}, x_{\alpha}, x_{\beta}\right)\right],
$$

where

$$
J_{i j \alpha \beta}^{M} \equiv \operatorname{Im}\left(U_{\alpha j} U_{\beta j} U_{\beta i}^{*} U_{\alpha i}^{*}\right) \quad \text { and } \quad J_{i j \alpha \beta}^{D} \equiv \operatorname{Im}\left(U_{\alpha j} U_{\beta j}^{*} U_{\beta i} U_{\alpha i}^{*}\right),
$$

are the phase factors obtained from the relevant Majorana and Dirac type diagrams. The dimensionless loop functions $I_{M}$ and $I_{D}$ are expressed in terms of the variables $x_{A} \equiv$ $m_{A}^{2} / m_{W}^{2}(A=i, j, \alpha, \beta)$; the loop functions of the dominant part are given in the appendix. The first term in eq. (3.8) comes from the diagrams (a1), (a2), (a3), (b1) and (b2), while the second term arises from the (c1) and (c2) diagrams. Notice that the loop function $I_{M}$ is always proportional to $m_{i} m_{j} / m_{W}^{2}$ because of the nature of the Majorana neutrinos. One can also see from the definition in eq. (3.9) that the phase factors $J_{i j \alpha \beta}^{M}$ and $J_{i j \alpha \beta}^{D}$ are anti-symmetric under the exchange of $i \leftrightarrow j$. As a result, only the anti-symmetric part of the loop functions $I_{M}$ and $I_{D}$ is relevant.

Since the loop function $I_{M}$ is always proportional to $m_{i} m_{j} / m_{W}^{2}$, it is convenient to extract this ratio as an overall factor. Moreover since the charged lepton mass is much lighter than the $W$ gauge boson mass, we can take $x_{\alpha}, x_{\beta} \approx 0$ as a good approximation if $m_{i}, m_{j} \gg m_{\alpha}, m_{\beta}\left(i, j>3\right.$ since, as one can see below, when the masses $m_{i}$ and $m_{j}$ are lighter than the charged lepton masses, the contributions to EDMs due to such light sterile states are extremely small). For all these reasons, eq. (3.8) can be simplified to

$$
\begin{aligned}
d_{\alpha} & =-\frac{g_{2}^{4} \text { e } m_{\alpha}}{4(4 \pi)^{4} m_{W}^{2}} \sum_{\beta} \sum_{i, j} \sqrt{x_{i} x_{j}}\left[J_{i j \alpha \beta}^{M} I_{M}^{\prime}\left(x_{i}, x_{j}, x_{\alpha}, x_{\beta}\right)+J_{i j \alpha \beta}^{D} I_{D}^{\prime}\left(x_{i}, x_{j}, x_{\alpha}, x_{\beta}\right)\right] \\
& \approx-\frac{g_{2}^{4} \text { e } m_{\alpha}}{4(4 \pi)^{4} m_{W}^{2}} \sum_{\beta} \sum_{i, j} \sqrt{x_{i} x_{j}}\left[J_{i j \alpha \beta}^{M} I_{M}^{\prime}\left(x_{i}, x_{j}\right)+J_{i j \alpha \beta}^{D} I_{D}^{\prime}\left(x_{i}, x_{j}\right)\right]
\end{aligned}
$$

where the relations among the loop functions are simply given by

$$
I_{M, D}=\sqrt{x_{i} x_{j}} I_{M, D}^{\prime} \text {, and } I_{M, D}^{\prime}\left(x_{i}, x_{j}\right) \equiv I_{M, D}^{\prime}\left(x_{i}, x_{j}, 0,0\right) .
$$




\section{$3.3 \quad 3+1$ model}

As a first step, we consider the minimal extension of the SM with one sterile fermion state, the $3+1$ model, which can be seen as the simplest effective model mimicking extensions of the SM accounting for neutrino data. Since the active neutrino masses and the charged lepton masses are much smaller than the $W$ boson mass, the loop functions are expanded to first order in terms of $x_{\beta=e, \mu, \tau}$ and $x_{i=1,2,3}$. Taking into account the fact that $\sum_{i} U_{\alpha i}^{*} m_{i} U_{\beta i}^{*}=0$ holds, one has in the $3+1$ model

$$
\sum_{i=1}^{3} \sqrt{x_{i}} J_{i 4 \alpha \beta}^{M}=0
$$

and the EDM formula of eq. (3.10) reduces to

$$
d_{\alpha} \approx-\frac{g_{2}^{4} e m_{\alpha}}{2(4 \pi)^{4} m_{W}^{2}} \sum_{\beta} \sum_{i=1}^{3} \sqrt{x_{i} x_{4}}\left[J_{i 4 \alpha \beta}^{M} x_{i} \frac{\partial I_{M}^{\prime}}{\partial x_{i}}\left(0, x_{4}\right)+J_{i 4 \alpha \beta}^{D} I_{D}^{\prime}\left(0, x_{4}\right)\right] .
$$

The first term in eq. (3.13) is highly suppressed by the factor $x_{i}=m_{i}^{2} / m_{W}^{2} \sim 10^{-24}$ for $m_{i} \sim 0.1 \mathrm{eV}$ (notice that the sum over $i$ runs over the three light - mostly active neutrino masses) and thus the EDM is dominated by the second term. Nevertheless, we have numerically checked that this latter term gives a negligible contribution to the EDMs. For instance, assuming $\mathcal{O}(1)$ loop functions and the mass of the sterile fermion state, e.g., $m_{4} \sim m_{W}$, the predicted EDM for the tau charged lepton is $\left|d_{\tau}\right| / e \lesssim 10^{-35} \mathrm{~cm}$. The EDMs for the electron and the muon are even smaller since the EDM is proportional to the charged lepton mass (see eq. (3.13)). Therefore, one can conclude that in the simple extension with one sterile neutrino, the predicted EDMs for the charged leptons are far below any future sensitivity. We thus consider the next to minimal extension of the SM by two sterile fermion states with masses $m_{4}$ and $m_{5}$.

\section{$3.43+2$ model}

The $3+2$ model is considered here as the next simplest effective model, where the masses of the two additional sterile states are greater that the ones of the active neutrinos. Taking this into account, and the fact that the phase factors and the loop functions are both anti-symmetric under the exchange $i \leftrightarrow j$ (see the discussion in section 3.2.2), the EDM formula in eq. (3.10) can be reduced as follows

$$
d_{\alpha} \approx-\frac{g_{2}^{4} \text { e } m_{\alpha}}{2(4 \pi)^{4} m_{W}^{2}} \sqrt{x_{4} x_{5}}\left[J_{\alpha}^{M} I_{M}^{\prime}\left(x_{4}, x_{5}\right)+J_{\alpha}^{D} I_{D}^{\prime}\left(x_{4}, x_{5}\right)\right]
$$

where $J_{\alpha}^{M, D}$ is defined by

$$
J_{\alpha}^{M, D} \equiv \sum_{\beta} J_{45 \alpha \beta}^{M, D}
$$

From this formula of eq. (3.14), one can see that the predicted contribution to the EDM has the potential to be large in this model if the scale of the two sterile neutrino masses is larger than the electroweak one $\left(x_{4,5} \gtrsim 1\right)$. The expressions for the reduced loop functions 
$I_{M}^{\prime}$ and $I_{D}^{\prime}$ (cf. eq. (3.11)) are obtained for the $3+2$ model; the expressions of the dominant parts are provided in the appendix.

If one considers further additional sterile fermion states, all the above discussion remains valid and we expect larger values for the charged lepton EDMs when the extra sterile states are heavier than the $W$ gauge boson mass.

In the numerical analysis we will consider this minimal scenario with only two sterile neutrinos (taking their masses, their active-sterile mixing angles and all the $\mathrm{CP}$-violating phases as free parameters).

\section{Constraints}

The modifications of the vertices in eq. (2.1) due to the presence of the rectangular $3 \times(3+2)$ leptonic mixing matrix imply deviations from unitarity of the $(3 \times 3)$ PMNS mixing matrix; moreover having massive sterile neutrinos as final decay products can possibly induce further deviations from the SM theoretical expectations. Consequently, scenarios with sterile fermions are severely constrained by numerous observables and bounds, among them EW precision measurements, charged lepton flavour violating observables and collider data.

In the following we address the most stringent constraints on the $3+2$ model. We focus on sterile neutrinos with masses above the GeV since the charged lepton EDMs roughly increase with the sterile neutrino mass as we have discussed in the previous section.

\subsection{Lepton flavour violating processes}

Charged lepton flavour violating processes such as $\mu \rightarrow e \gamma$ and $\mu \rightarrow e \bar{e} e$ give strong constraints on the parameter space. The radiative process $\mu \rightarrow e \gamma$ is induced at the one-loop level, and its branching ratio is computed as [71]

$$
\operatorname{Br}(\mu \rightarrow e \gamma)=\frac{\sqrt{2} G_{F}^{2} m_{\mu}^{5}}{\Gamma_{\mu}}\left|\sum_{i=4}^{3+N} U_{\alpha i} U_{\beta i}^{*} G_{\gamma}\left(\frac{m_{i}^{2}}{m_{W}^{2}}\right)\right|^{2}
$$

where $G_{F}$ is the Fermi constant, $\Gamma_{\mu}$ is the muon total decay width given by [55]

$$
\Gamma_{\mu}=\frac{G_{F}^{2} m_{\mu}^{5}}{192 \pi^{3}}\left(1-8 \frac{m_{e}^{2}}{m_{\mu}^{2}}\right)\left[1+\frac{\alpha_{\mathrm{em}}}{2 \pi}\left(\frac{25}{4}-\pi^{2}\right)\right]
$$

and the loop function $G_{\gamma}(x)$ is given by [55]

$$
G_{\gamma}(x)=\frac{x-6 x^{2}+3 x^{3}+2 x^{4}-6 x^{3} \log x}{4(1-x)^{4}} .
$$

The current experimental bound for this process is $\operatorname{Br}(\mu \rightarrow e \gamma) \leq 5.7 \times 10^{-13}[72]$, and the expected future sensitivity by the upgraded MEG experiment is $\operatorname{Br}(\mu \rightarrow e \gamma) \sim 6 \times 10^{-14}$ [73]. 
Another cLFV process, $\mu \rightarrow e \bar{e} e$, is also induced at the one-loop level and its branching ratio is given by [55]

$$
\begin{aligned}
\operatorname{Br}(\mu \rightarrow e \bar{e} e)= & \frac{G_{F}^{4} m_{W}^{4} m_{\mu}^{5}}{6144 \pi^{7} \Gamma_{\mu}}\left[2\left|\frac{1}{2} F_{\mathrm{Box}}^{\mu e e e}+F_{Z}^{\mu e}-2 \sin ^{2} \theta_{W}\left(F_{Z}^{\mu e}-F_{\gamma}^{\mu e}\right)\right|^{2}\right. \\
& +4 \sin ^{4} \theta_{W}\left|F_{Z}^{\mu e}-F_{\gamma}^{\mu e}\right|^{2}+16 \sin ^{2} \theta_{W} \operatorname{Re}\left\{\left(F_{Z}^{\mu e}+\frac{1}{2} F_{\mathrm{Box}}^{\mu e e e}\right) G_{\gamma}^{\mu e *}\right\} \\
& \left.-48 \sin ^{4} \theta_{W} \operatorname{Re}\left\{\left(F_{Z}^{\mu e}-F_{\gamma}^{\mu e}\right) G_{\gamma}^{\mu e *}\right\}+32 \sin ^{4} \theta_{W}\left|G_{\gamma}^{\mu e}\right|^{2}\left\{\log \frac{m_{\mu}^{2}}{m_{e}^{2}}-\frac{11}{4}\right\}\right],
\end{aligned}
$$

where the relevant loop functions $F_{\mathrm{Box}}^{\mu e e e}, F_{Z}^{\mu e}, F_{\gamma}^{\mu e}$ and $G_{\gamma}^{\mu e}$ are given in ref. [56]. The experimental bound for this process is $\operatorname{Br}(\mu \rightarrow e \bar{e} e) \leq 1.0 \times 10^{-12}$ [74]. According to the research proposal of the Mu3e experiment, the sensitivity will reach $\operatorname{Br}(\mu \rightarrow e \bar{e} e) \sim 10^{-16}$ [41].

While the other constraints, which will be discussed below, are mainly related to the mixings $\left|U_{\alpha i}\right|^{2}$, the cLFV processes constrain combinations like $\left|U_{\alpha i} U_{\beta i}^{*}\right|$. In our analysis, we have computed the above mentioned observables in the $3+2$ model.

\subsection{Direct collider production}

When the sterile neutrino mass is $m_{i} \lesssim \mathcal{O}(100) \mathrm{GeV}$, a strong constraint is given by the LEP experiment. The relevant process is $e^{+} e^{-} \rightarrow \nu_{i} \nu_{j}^{*} \rightarrow \nu_{i} e^{ \pm} W^{\mp}$ where $i \leq 3$ and $j \geq 4$ and it violates lepton number conservation due to Majorana neutrinos. Hence, certain regimes of the mixing angles $\left|U_{\alpha i}\right|$ are already excluded by LEP data [42].

The other bound is given by LHC data for $m_{i} \gtrsim \mathcal{O}(100 \mathrm{GeV})$ searching for a same sign di-lepton channel $p p \rightarrow W^{ \pm *} \rightarrow \ell^{ \pm} \nu_{i} \rightarrow \ell^{ \pm} \ell^{ \pm} j j$ where $i \geq 4$ and $j$ denotes a jet. With an integrated luminosity of $20 \mathrm{fb}^{-1}$ at $\sqrt{s}=8 \mathrm{TeV}$, LHC data allows to constrain [36, 37] the mixing angle $\left|U_{\alpha i}\right|$ for sterile neutrino masses up to $500 \mathrm{GeV}$.

As future prospects, the $\sqrt{s}=14 \mathrm{TeV}$ LHC [36, 38] and Future Lepton Colliders (like ILC) $[46,47]$ are expected to give a stronger bound on the mixing angles. The collider bounds discussed here including the other constraints have been investigated in detail in refs. [42-44].

\subsection{Electroweak precision data}

The active-sterile mixings affect electroweak precision observables such as the $W$ boson decay width, the $Z$ invisible decay, meson decays and the non-unitarity of the $3 \times 3$ submatrix ( $\left.U_{\mathrm{PMNS}}\right)$ of $U_{i j}$.

The constraints on the $W$ decay and $Z$ invisible decay are mostly relevant for $m_{i}<$ $m_{W}, m_{Z}$, respectively. The constraints given by DELPHI [75] and L3 [76] Collaborations are the strongest for the sterile neutrino mass range $3 \mathrm{GeV} \lesssim m_{i} \lesssim 90 \mathrm{GeV}$. A future high luminosity $Z$ factory, such as FCC-ee, will give significant improvements for the constraints in this mass range $[77,78]$.

The existence of sterile neutrinos may also violate lepton flavour universality of meson decays such as $\pi^{+} \rightarrow \ell_{\alpha}^{+} \nu_{\alpha}$ and $K^{+} \rightarrow \ell_{\alpha}^{+} \nu_{\alpha}$ [79-81]. In particular, when the sterile neutrino 
mass is below the threshold $m_{i} \leq m_{\pi^{+}}, m_{K^{+}}$, the meson decay gives a strong constraint on the mixing matrix $U_{\alpha i}$ where $m_{\pi^{+}}=139.6 \mathrm{MeV}$ and $m_{K^{+}}=493.7 \mathrm{MeV}$.

The non-unitarity of the $U_{\mathrm{PMNS}}$ sub-matrix is constrained by some experiments such as the above electroweak precision data and cLFV processes. The constraints for each component of the sub-matrix have been discussed in refs. [82, 83].

\subsection{Perturbative unitarity bound}

Any coupling of the sterile fermions to the SM particles must be perturbative; in particular, all the couplings in eq. (2.1) should be perturbative. If the additional two sterile fermion states are heavy enough to decay into a $W$ boson and a charged lepton, or into an active neutrino and either a $Z$ or a Higgs boson, their decay widths should comply with the perturbative unitarity condition [78, 84-89]. In this case, since the dominant decay mode of the sterile neutrinos $\nu_{4,5}$ would be $\nu_{i} \rightarrow \ell_{\alpha}^{\mp} W^{ \pm}$, the decay width of $\nu_{i}(i=4,5)$ has to comply with the perturbative unitary bound: ${ }^{5}$

$$
\frac{\Gamma_{\nu_{i}}}{m_{i}}<\frac{1}{2} \quad \text { where } \quad \Gamma_{\nu_{i}} \approx \frac{g_{2}^{2} m_{i}^{3}}{16 \pi m_{W}^{2}} \sum_{\alpha}\left|U_{\alpha i}\right|^{2},(i=4,5),
$$

which translates into an upper bound on the sterile neutrino masses as follows,

$$
m_{i} \lesssim 873 \mathrm{GeV}\left(\sum_{\alpha}\left|U_{\alpha i}\right|^{2}\right)^{-1 / 2} .
$$

\subsection{Other constraints}

Cosmological observations, see for instance ref. [34], put severe constraints on sterile neutrinos with a mass below the $\mathrm{GeV}$ scale. Since, and as already discussed, the contributions of the sterile fermion states to charged lepton EDMs are negligible for masses below the EW scale, we do not apply constraints from Big Bang Nucleosysthesis or Cosmic Microwave Background, which would be relevant in the very low mass regime. For heavier mass regimes, the perturbative unitarity condition of eq. (4.6) turns out to be also very constraining.

The additional sterile fermions might contribute to neutrinoless double beta decay, and the corresponding effective mass $m_{e e}$ is corrected according to [90, 91]

$$
m_{e e} \simeq \sum_{i=1}^{5} U_{e i}^{2} p^{2} \frac{m_{i}}{p^{2}-m_{i}^{2}},
$$

where $p^{2} \simeq-(125 \mathrm{MeV})^{2}$ is the virtual momentum of the neutrino. Several experiments (among them GERDA [92], EXO-200 [93, 94], KamLAND-ZEN [95]) have put constraints on the effective mass, which translate into bounds on combinations of $U_{e i}^{2} m_{i}, i=4,5$. In our numerical analysis, we have checked that our solutions always comply with the conservative experimental bound $\left|m_{e e}\right| \lesssim 0.01 \mathrm{eV}$ (since the cancellation between each $i$-th contribution can occur due to the existence of the CP-violating phases).

\footnotetext{
${ }^{5}$ Another common criterion of perturbativity is that the couplings should be less than $\sqrt{4 \pi}$. This criterion also gives a bound similar to eq. (4.6).
} 


\section{$5 \quad$ Numerical results}

We now proceed with the numerical evaluation of the EDMs of charged leptons in the $3+2$ model. As described in section 2, this simple model allows to illustrate the potential effects of the addition of the number $N>2$ of sterile fermion states. For completeness, we also address the muon magnetic dipole moment, the first non-vanishing contribution arising at the one-loop level.

In the numerical analysis we have conducted, we consider the minimal scenario with only two sterile neutrinos, taking their masses, their active-sterile mixing angles and all the CP-violating phases as free parameters.

\subsection{Electric dipole moments of charged leptons}

In order to investigate the parameter space of the $3+2$ model for the charged lepton EDMs, we vary the parameters in the following ranges:

$$
1 \mathrm{GeV} \leq m_{i} \leq 10^{6} \mathrm{GeV}, \quad \sin \theta_{j i} \leq 0.1, \text { for } j=1,2,3 \text { and } i=4,5, \quad \sin \theta_{45} \leq 1,
$$

and all the Dirac and Majorana CP-violating phases are taken in the range of $[0,2 \pi]$. The mixing between the two sterile states, $\theta_{45}$, is not constrained.

The reduced loop functions $I_{M}^{\prime}$ and $I_{D}^{\prime}$, defined in eq. (3.14), and whose analytical expressions of the dominant parts are given in the appendix, have been numerically evaluated. To illustrate their relative contributions (their coefficients, namely the phase factors $J_{\alpha}^{M, D}$, saturating at 1 ), we display on figure 3 the loop integrals $I_{M}^{\prime}$ and $I_{D}^{\prime}$ as a function of $m_{4}$ for several fixed values of $m_{5}$. One can see that the loop function $I_{D}^{\prime}$ is $\mathcal{O}(1)$ in most of the considered mass range, while the loop function $I_{M}^{\prime}$ increases logarithmically with $x_{4}$. Due to this, the Majorana contribution $J_{\alpha}^{M} I_{M}^{\prime}$ in eq. (3.14) gives the dominant contribution to the EDMs in most of the considered mass range, independently of the active-sterile mixings.

On figure 4 we display the allowed parameter space for the phase factors $\left|J_{\alpha}^{M}\right|$ and $\left|J_{\alpha}^{D}\right|$ defined in eqs. (3.9), (3.15), after having applied all the constraints discussed in section 4. As one can see, in each of the 3 panels corresponding to the three charged leptons, the upper right region is excluded by the perturbative unitarity constraint. The region corresponding to $m_{i} \lesssim 70 \mathrm{GeV}$ is strongly constrained by LEP. Constraints from electroweak precision data are also important and almost independent of the sterile neutrino mass when $m_{i} \gtrsim 1 \mathrm{GeV}$. Finally, the region which would lead to $\left|J_{\alpha}^{M}\right|,\left|J_{\alpha}^{D}\right| \gtrsim 10^{-6}$ is constrained by the bounds on cLFV processes. As one can see from this figure, there is no substantial difference between $\left|J_{\alpha}^{M}\right|$ and $\left|J_{\alpha}^{D}\right|$. Moreover, the allowed region for all the charged leptons is almost the same. Therefore, using eq. (3.14), an approximate relation among the charged lepton EDMs is found as

$$
\frac{\left|d_{e}\right|}{m_{e}} \sim \frac{\left|d_{\mu}\right|}{m_{\mu}} \sim \frac{\left|d_{\tau}\right|}{m_{\tau}} .
$$

For the theoretically and experimentally viable regions of the parameter space, the charged lepton EDMs are shown in figure 5 as a function of $\theta_{i 4}, i=1,2,3$ (left panels) 

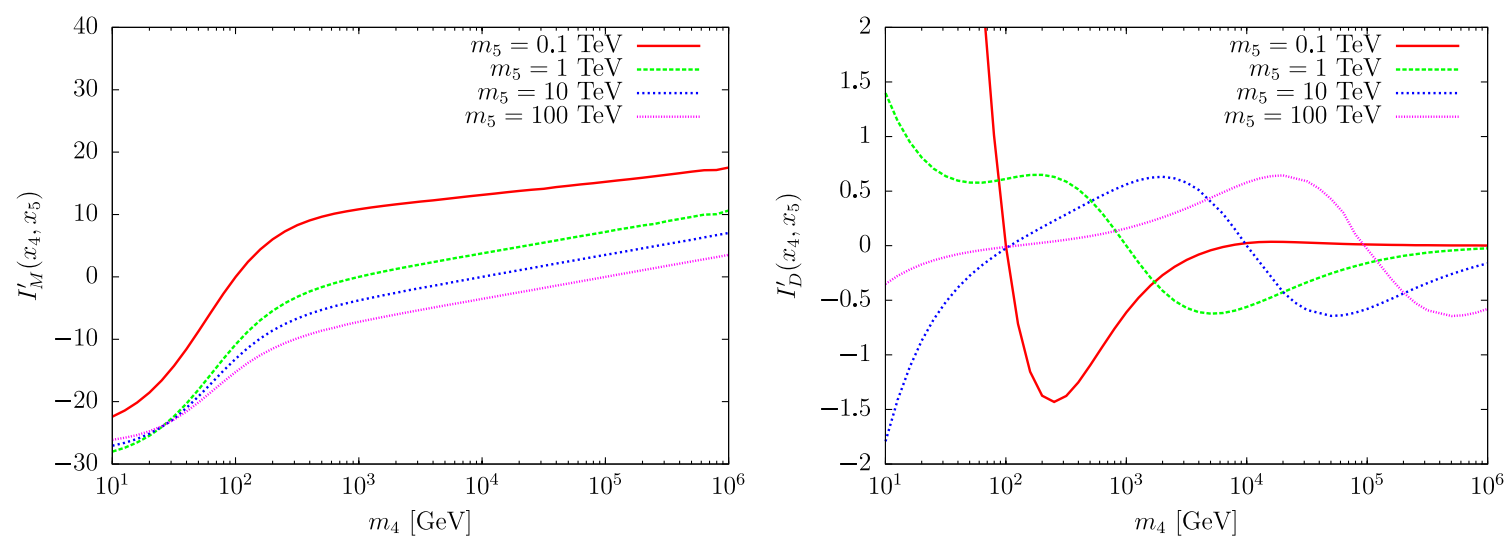

Figure 3. Loop functions $I_{M}^{\prime}$ and $I_{D}^{\prime}$ as a function of $m_{4}$ for several fixed values of $m_{5}$.
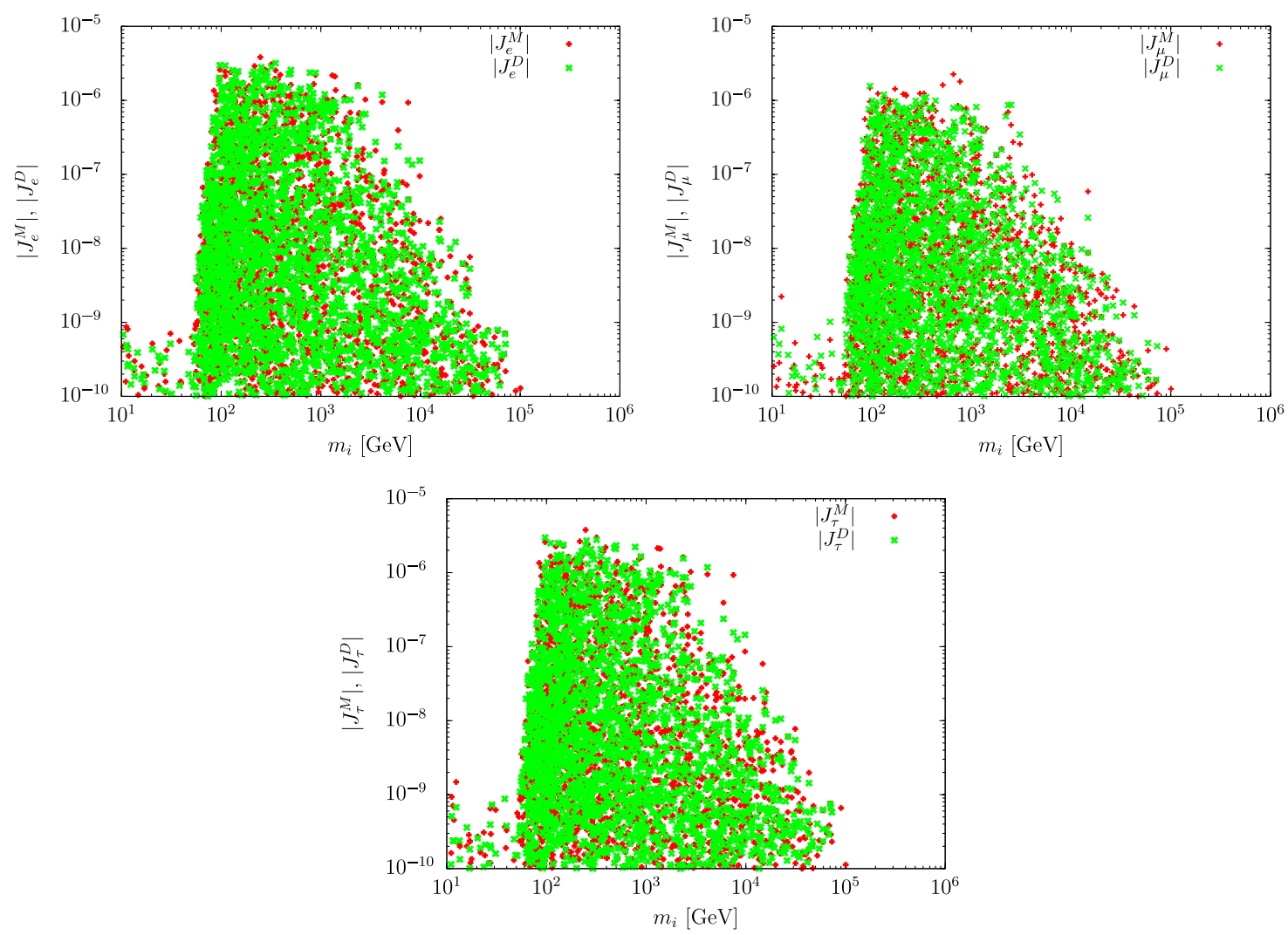

Figure 4. Phase factors $\left|J_{\alpha}^{M}\right|$ and $\left|J_{\alpha}^{D}\right|$ as a function of $m_{i}$, where $J_{\alpha}^{M, D}=\sum_{\beta} J_{45 \alpha \beta}^{M, D}$ for the three charged leptons.

and of $m_{4}$ (right panels), respectively. The green points comply with all the imposed constraints while the red points are excluded by the bounds on cLFV processes. The current experimental bounds and future sensitivities are also shown as blue and black lines. Analogous results would be obtained when displaying these observables as a function of $\theta_{i 5}$ and $m_{5}$, as the two sterile states play similar rôles in the several diagrams. As one 

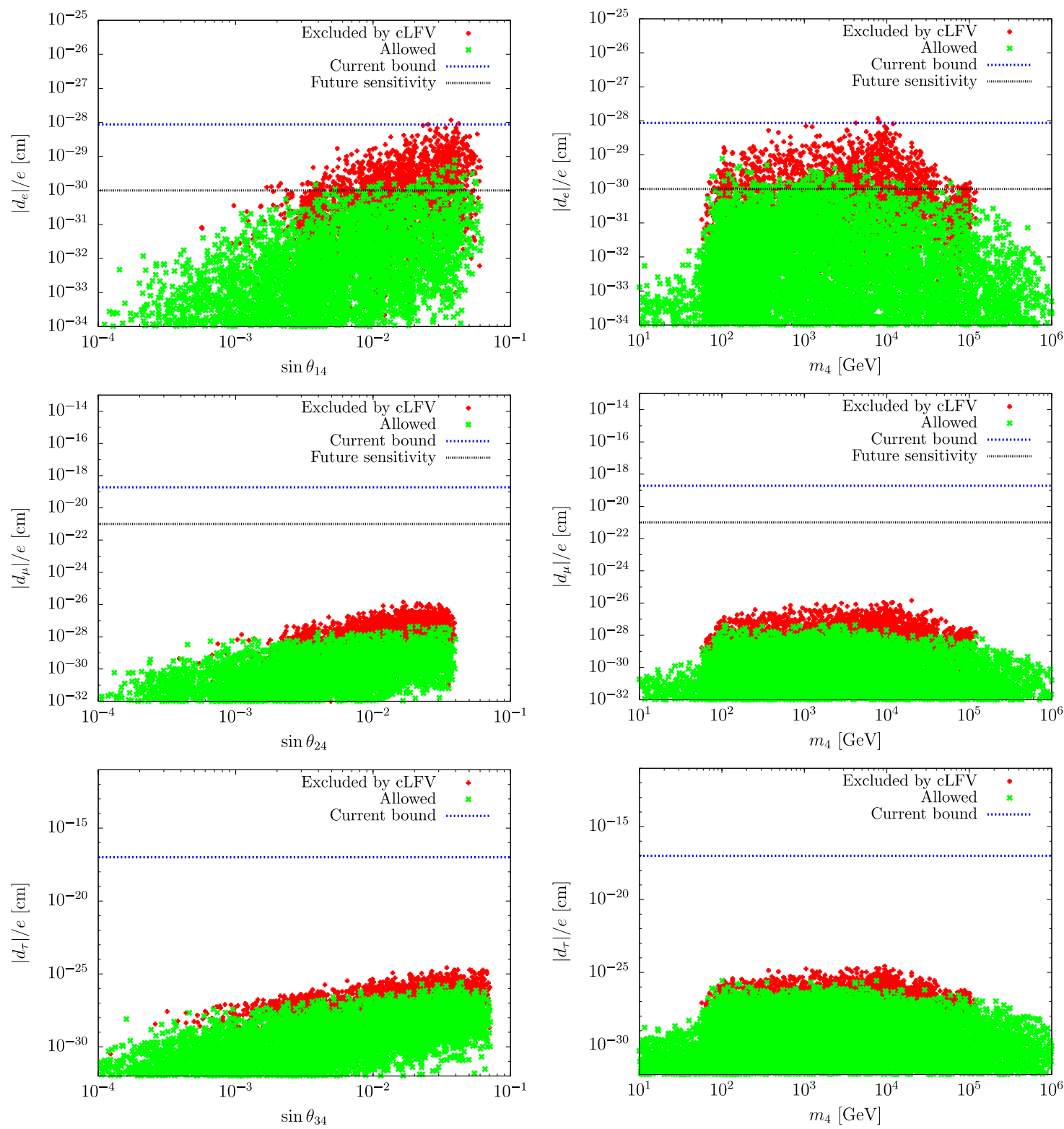

Figure 5. Charged lepton EDMs in the $3+2$ effective model as a function of $\theta_{i 4}, i=1,2,3$ (left) and $m_{4}$ (right). The current upper bounds and future prospects are also shown as blue and black lines respectively.

can see from figure 5, the maximum values for the EDMs are obtained in the ranges of $\sin \theta_{i 4} \gtrsim 10^{-2}(i=1,2,3)$ and $100 \mathrm{GeV} \lesssim m_{4} \lesssim 100 \mathrm{TeV}$. The range $\sin \theta_{i 4} \gtrsim 0.1$ is excluded by the constraints discussed in the previous section, in particular by electroweak precision data. The electron EDM is always below the current experimental upper bound $\left|d_{e}\right| / e<8.7 \times 10^{-29} \mathrm{~cm}$ (so no additional bound on the parameter space arises from this CP-violating observable), but the corresponding contributions can be within the optimistic future sensitivity, $\left|d_{e}\right| / e=10^{-30} \mathrm{~cm}$. On the other hand for the muon and tau, the predicted EDMs are much smaller than any future sensitivity. 
One can also try to investigate which combinations of the 10 physical CP-violating phases are relevant for the EDMs; however, this task is very involved and we have not identified significant correlations between a given phase (or combination of phases) in our parametrisation and the contributions to the EDMs.

To conclude the discussion of the charged lepton EDMs, we present in figure 6 the predictions for the electron EDM in the $\left(\left|U_{\alpha i}\right|^{2}, m_{i}\right)(\alpha=e, \mu, \tau, i=4,5)$ parameter space of the $3+2$ model. Coloured surfaces reflect the violation of at least one phenomenological or cosmological bound as presented in section 4 (see also the discussion of $[42,44,48]$ ). The different lines correspond to the reach of future facilities: the projected exclusion limit from the LHC (14 TeV run data [42]), the expected sensitivity of FCC-ee regarding the production of heavy sterile neutrinos [77], DUNE [39] and SHiP (a fixed-target experiment using high-intensity proton beams at the CERN SPS [40, 45]). The displayed green points correspond to having the electron EDM larger than the future sensitivity, i.e. $\left|d_{e}\right| / e>10^{-30}$.

As one can see on the first panel of figure 6 , in view of the associated large regime for the active-sterile mixing $\left|U_{e i}\right|^{2}$, some points can be tested by future collider experiments such as the LHC with $\sqrt{s}=14 \mathrm{TeV}$ and the future ILC. Despite their impressive sensitivities, future experiments such as LBNE, SHiP and FCC-ee will not be able to probe the regions in parameter space responsible for sizable EDM contributions, since these facilities aim at sterile mass regimes below the EW scale (recall that in order to have the electron EDM within experimental sensitivity, the sterile masses should be $100 \mathrm{GeV} \lesssim m_{i} \lesssim 100 \mathrm{TeV}$ ).

\subsection{Muon anomalous magnetic moment}

As already mentioned, sterile fermion states can have an impact on CP- and flavourconserving observables. Here, and for completeness, we briefly address the impact of the 2 extra sterile states on the muon anomalous magnetic moment. The current experimental value of the muon anomalous magnetic moment has been measured by Muon $g-2$ Collaboration [96], and the discrepancy of the muon anomalous magnetic moment between the experimental value and the SM prediction is given by [60]

$$
\Delta a_{\mu} \equiv a_{\mu}^{\exp }-a_{\mu}^{\mathrm{SM}}=2.88 \times 10^{-9} .
$$

In our scenario, the muon anomalous magnetic moment induced by the $W$ boson and neutrino loop can be computed at one-loop level as [91]

$$
a_{\mu}=\frac{\sqrt{2} G_{F} m_{\mu}^{2}}{(4 \pi)^{2}} \sum_{i=1}^{3+N}\left|U_{\mu i}\right|^{2} F\left(\frac{m_{i}^{2}}{m_{W}^{2}}\right)
$$

where the loop function $F(x)$ is defined by

$$
F(x)=\frac{10-43 x+78 x^{2}-49 x^{3}+4 x^{4}+18 x^{3} \log x}{3(1-x)^{4}} .
$$



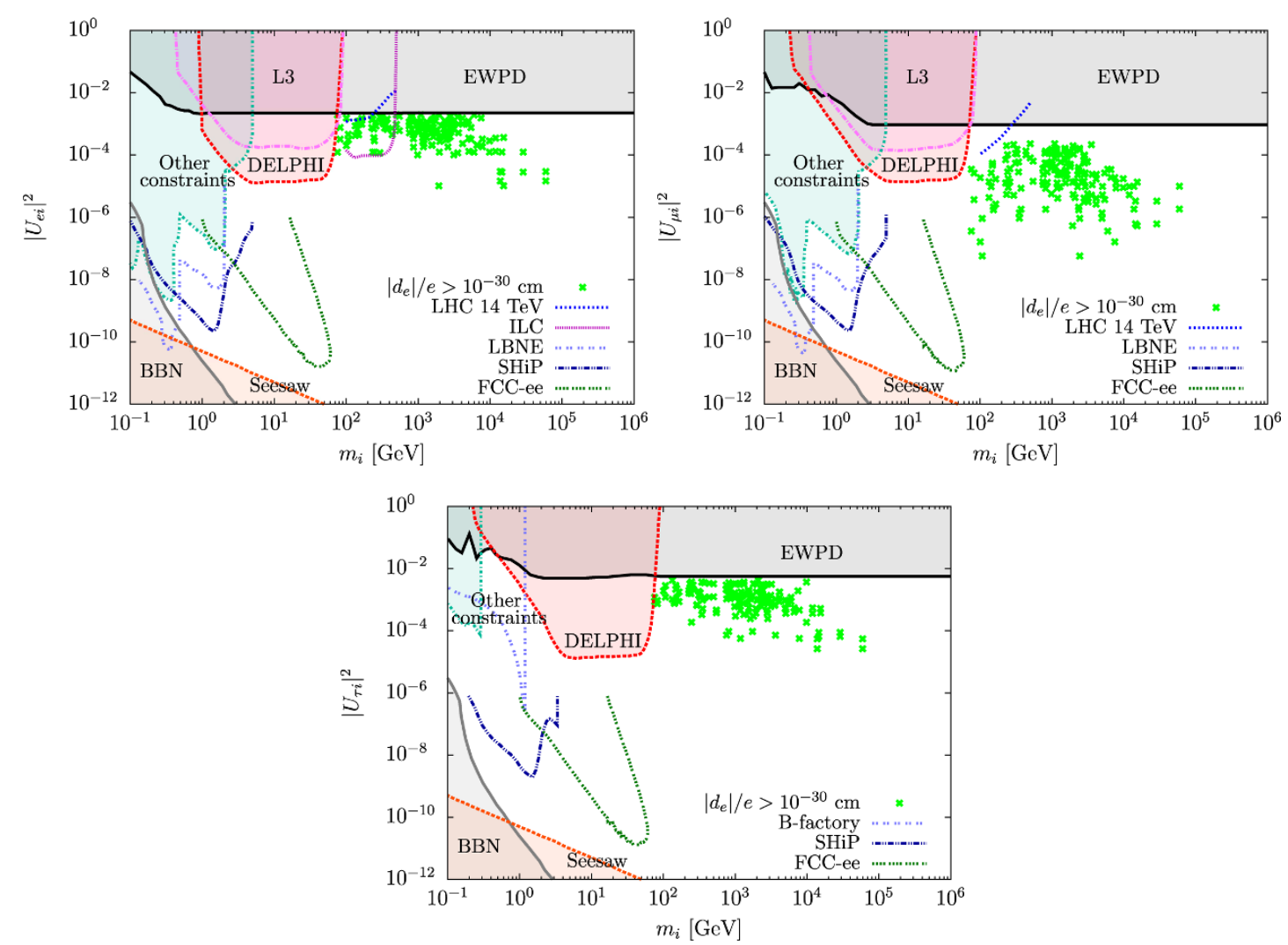

Figure 6. Parameter spaces $\left(\left|U_{\alpha i}\right|^{2}, m_{i}\right)$, for $\alpha=e, \mu, \tau$ and $i=4$ or 5 . The coloured surfaces are excluded due to the violation of at least one experimental or observational -mostly from BBNbound. Lines (full, dashed and dotted) delimit the expected sensitivity of several facilities: DUNE, SHiP, FCC-ee and LHC. Green points denote predictions for the electron EDM within the future sensitivity reach, $\left|d_{e}\right| / e \geq 10^{-30} \mathrm{~cm}$.

Subtracting the (one-loop with mostly active neutrino contributing in the loop) SM contribution in eq. (5.4), one obtains

$$
\Delta a_{\mu} \approx-\frac{4 \sqrt{2} G_{F} m_{\mu}^{2}}{(4 \pi)^{2}} \sum_{i=4}^{3+N}\left|U_{\mu i}\right|^{2} G_{\gamma}\left(\frac{m_{i}^{2}}{m_{W}^{2}}\right)
$$

where the active neutrino masses $m_{i}(i=1,2,3)$ are neglected and $G_{\gamma}(z)$ is defined by eq. (4.3). As has been shown in [91], the new contribution to the muon anomalous magnetic moment can hardly fill the unexplained discrepancy with experiment. Taking into account all the experimental constraints discussed in sections 4 and 5 , the predicted value of the muon anomalous magnetic moment is roughly $\Delta a_{\mu} \sim-10^{-12}$ for $\left|U_{\mu i}\right|^{2} \sim 10^{-3}$, and thus additional contributions to the anomalous magnetic moment are still required to explain the discrepancy between theory and experimental measurements. 


\section{Summary}

We have discussed the contributions of sterile neutrinos to charged lepton EDMs in the SM minimally extended via $N$ sterile fermion states. We have considered all the diagrams which can potentially contribute to charged lepton EDMs. The comparison of the different contributions has shown that significant contributions to the charged lepton EDMs can only be obtained if the (sterile) neutrinos are of Majorana nature.

In the case of the $3+1(N=1)$ model, we have found that the predicted EDMs are too small to be detected in any foreseen future experiments. We have moreover verified that at least two sterile neutrinos are required to obtain an electron EDM within future sensitivity reach. In this most minimal scenario $(N=2)$, the masses of the two sterile states should be in the range $100 \mathrm{GeV}-100 \mathrm{TeV}$ to have $\left|d_{e}\right| / e \geq 10^{-30} \mathrm{~cm}$. For the muon and the tau, the predicted EDMs remain several orders of magnitude below the future sensitivities.

In our analysis we imposed all available experimental and observational constraints on sterile neutrinos, and we also discussed the prospects of probing this scenario at low and high energy experiments. In particular, regions in parameter space which predict a large electron EDM could be also explored by collider experiments such as a Future Linear Collider (ILC) and marginally with the $\sqrt{s}=14 \mathrm{TeV}$ LHC.

\section{Acknowledgments}

The authors would like to thank Michele Lucente and Olcyr Sumensari for fruitful discussions. A.A. is grateful to Amon Ilakovac and to Avelino Vicente for useful discussions. We acknowledge support from the European ITN project (FP7-PEOPLE-2011-ITN, PITNGA-2011-289442-INVISIBLES). T.T. acknowledges support from P2IO Excellence Laboratory (LABEX). This work was done in the framework of a "Défi InPhyNiTi" project (N2P2M-SF)

\section{A Loop calculations}

In what follows we provide the leading terms of the loop functions in the limit where $x_{i}, x_{j} \gg 1$. When the sterile neutrino masses are much heavier than the $W$ boson mass $\left(x_{i}, x_{j} \gg 1\right)$, the loop functions satisfy the relation $I_{M}^{\prime}\left(x_{i}, x_{j}\right) \gg I_{D}^{\prime}\left(x_{i}, x_{j}\right)$ due to the additional factor $\sqrt{x_{i} x_{j}}$ for $I_{M}^{\prime}\left(x_{i}, x_{j}\right)$ where $I_{M, D}^{\prime}\left(x_{i}, x_{j}\right)$ is defined in eq. (3.10). Moreover, the loop function $I_{M}^{\prime}\left(x_{i}, x_{j}\right)$ contains terms increasing with $\log \left(x_{i} / x_{j}\right)$, which turn out to be the dominant ones.

In general the loop function $I_{M}^{\prime}\left(x_{i}, x_{j}\right)$ can be expressed by anti-symmetrizing in terms of $x_{i}$ and of $x_{j}$ as

$$
I_{M}^{\prime}\left(x_{i}, x_{j}\right)=\frac{1}{2}\left(I_{M 0}^{\prime}\left(x_{i}, x_{j}\right)-I_{M 0}^{\prime}\left(x_{j}, x_{i}\right)\right),
$$

where $I_{M 0}^{\prime}\left(x_{i}, x_{j}\right)$ is given by

$$
I_{M 0}^{\prime}\left(x_{i}, x_{j}\right)=I_{M 01}^{\prime(a)}\left(x_{i}, x_{j}\right)+I_{M 02}^{\prime(a)}\left(x_{i}, x_{j}\right)+I_{M 01}^{\prime(b)}\left(x_{i}, x_{j}\right)+I_{M 02}^{\prime(b)}\left(x_{i}, x_{j}\right),
$$

and the four functions in right-hand side in eq. (A.2) will be given below. The superscripts $(a),(b)$ denote contributions coming from the diagrams in the first and second lines in 
figure 1 respectively; the subscript 1,2 translates that two different functions exist for each diagram. The loop function $I_{M 0 n}^{\prime(a, b)}(n=1,2)$ for the diagrams in the first and second lines in figure 1 are given by

$$
\begin{aligned}
& I_{M 0 n}^{\prime(a)}\left(x_{i}, x_{j}\right)=\int_{0}^{1} \prod_{A=1}^{4} d s_{A} \delta\left(\sum_{A=1}^{4} s_{A}-1\right) \int_{0}^{1} \prod_{B=1}^{3+\delta_{2 n}} d t_{B} \delta\left(\sum_{B=1}^{3+\delta_{2 n}} t_{B}-1\right) F_{n}^{(a)}\left(x_{i}, x_{j}\right), \\
& I_{M 0 n}^{\prime(b)}\left(x_{i}, x_{j}\right)=\int_{0}^{1} \prod_{A=1}^{5} d s_{A} \delta\left(\sum_{A=1}^{5} s_{A}-1\right) \int_{0}^{1} \prod_{B=1}^{3+\delta_{2 n}} d t_{B} \delta\left(\sum_{B=1}^{3+\delta_{2 n}} t_{B}-1\right) F_{n}^{(b)}\left(x_{i}, x_{j}\right),
\end{aligned}
$$

where $F_{n}^{(a, b)}\left(x_{i}, x_{j}\right)$ is given by

$$
\begin{aligned}
& F_{n}^{(a)}\left(x_{i}, x_{j}\right)=\frac{N_{n}^{(a 1)}}{D^{(a 1)}}+\frac{N_{n}^{(a 2)}}{D^{(a 2)}}+\frac{N_{n}^{(a 3)}}{D^{(a 3)}}, \\
& F_{n}^{(b)}\left(x_{i}, x_{j}\right)=\frac{N_{n}^{(b 1)}}{D^{(b 1)}}+\frac{N_{n}^{(b 2)}}{D^{(b 2)}} .
\end{aligned}
$$

Each of the terms corresponds to the contribution coming from the diagrams (a1), (a2), (a3), (b1) and (b2). The denominators are given by

$$
\begin{aligned}
D^{(a 1)}= & D^{(a 2)}=s_{2}\left(s_{2}-1\right)\left(t_{1} x_{j}+t_{2}\right)-\left(1-t_{1}-t_{2}\right)\left(s_{1} x_{i}+s_{3}+s_{4}\right), \\
& D^{(a 3)}=\left(s_{2}+s_{3}\right)\left(s_{2}+s_{3}-1\right)\left(t_{1} x_{i}+t_{2}\right)-\left(1-t_{1}-t_{2}\right)\left(x_{1} x_{j}+x_{4}\right), \\
& D^{(b 1)}=-\left(1-t_{1}\right)\left(s_{1}+s_{4}+s_{5}+s_{2} x_{i}+s_{3} x_{j}\right), \\
& D^{(b 2)}=t_{1}\left(s_{4}+s_{5}\right)\left(s_{4}+s_{5}-1\right)-\left(1-t_{1}\right)\left(s_{1}+s_{2} x_{i}+s_{3} x_{j}\right),
\end{aligned}
$$

and the numerators for the diagram (a1), (a2), (a3) are given by

$$
\begin{aligned}
N_{1}^{(a 1)}+N_{1}^{(a 2)}= & 3\left(s_{1}-s_{2}\right) t_{2}+s_{1}\left(5 t_{1}+1\right) t_{3}+5 s_{2}\left(1-t_{1}\right) t_{2} \\
& -s_{1}-11 t_{2}-t_{3}+9+\frac{s_{1}\left(-5 t_{2}-5 t_{3}+13\right) t_{3}}{s_{2}-1} \\
& +\frac{x_{i}}{2} \frac{t_{3}\left(s_{3}+s_{4}\right)}{s_{2}-1}+\frac{x_{j}}{2}\left(\frac{5 s_{1} t_{3}}{s_{2}-1}-6\left(s_{1} t_{3}+s_{2} t_{2}-s_{1}\right)+t_{3}-2\right), \\
N_{1}^{(a 3)}= & -\left(5 s_{1}+1\right)\left(1-t_{1}\right)-\left(s_{1}+1\right) t_{2}+\left(s_{2}+s_{3}-2\right)\left(2 t_{1}-1\right) t_{2} \\
& +3 s_{1}+1+\frac{2 s_{1} s_{4} t_{3}^{2}}{\left(s_{2}+s_{3}-1\right)^{2}}+\frac{s_{1} t_{3}\left(-2 s_{4} t_{3}-2 s_{1}+1\right)}{s_{2}+s_{3}-1} \\
& +\frac{x_{i}}{2}\left(2\left(t_{2}-t_{3}\right)+3\left(t_{3}-1\right)\left(2 s_{1}+s_{2}+s_{3}\right)\right), \\
N_{2}^{(a 1)}+N_{2}^{(a 2)}= & -20 s_{2} t_{2}^{2}-2\left(s_{1}-s_{2}-12\right) t_{2}-4\left(5\left(t_{3}+t_{4}\right)-4\right)\left(s_{1} t_{1}+s_{2} t_{2}\right) \\
& +2 t_{1}-8+\frac{s_{1}\left(-8 t_{2}+4\left(10\left(1-t_{1}\right)-23\right)\left(t_{3}+t_{4}\right)+19\right)}{2\left(s_{2}-1\right)}+\frac{17 s_{1}-1}{2 s_{2}} \\
& +\frac{x_{i}}{2} \frac{s_{2}\left(-6 t_{2}\left(s_{2}-1\right)+s_{2}-1\right)+s_{1}\left(s_{2}\left(6 t_{3}+6 t_{4}-5\right)+4\right)}{\left.s_{2}-1\right) s_{2}} \\
& +\frac{x_{j}}{2} \frac{s_{1}\left(5-6 s_{2}\left(t_{1}+t_{2}\right)\right)-\left(s_{2}-1\right)\left(6 t_{2} s_{2}-1\right)}{(\mathrm{A} .13}
\end{aligned}
$$




$$
\begin{aligned}
N_{2}^{(a 3)}= & -\frac{2 s_{4}^{2}\left(4\left(t_{3}+t_{4}\right)^{2}-6\left(t_{3}+t_{4}\right)+1\right)}{-s_{1}-s_{4}}-8 s_{4} t_{3}^{2}-2 s_{4} t_{3}\left(8 t_{4}-7\right) \\
& -s_{4}\left(t_{4}-1\right)\left(8 t_{4}-3\right)-8\left(s_{1}+s_{4}\right) t_{2}^{2}+3 s_{1} t_{3}+3\left(s_{2}+s_{3}\right)\left(1-t_{4}\right) \\
& +\frac{2\left(s_{4}-2\right)\left(s_{4}\left(t_{3}-2\right)+1\right)-3 s_{1}\left(2 t_{2}+t_{3}\right)+\left(s_{4}-3\right)\left(2 s_{4}-1\right) t_{4}+9 t_{2}}{s_{2}+s_{3}} \\
& +t_{2}\left(-8\left(s_{1}+s_{4}\right)\left(t_{3}+t_{4}\right)+6 s_{1}-7\left(s_{2}+s_{3}\right)-2\right)+1 \\
& +\frac{x_{i}}{2}\left(3\left(t_{3}+t_{4}+1\right)\left(2 s_{4}+s_{3}+s_{2}\right)-3\left(t_{3}+t_{4}\right)-1\right) .
\end{aligned}
$$

Note that since the diagrams (a1) and (a2) give the same contribution, one has the relation $N_{n}^{(a 1)}=N_{n}^{(a 2)}$. The numerators for the diagrams (b1) and (b2) are given by

$$
\begin{aligned}
N_{1}^{(b 1)}= & \frac{3 s_{1}\left(t_{2}+t_{3}\right)\left(\left(s_{3}-s_{2}\right)\left(1+s_{4}+s_{5}\right)\left(t_{2}+t_{3}\right)+\left(2-s_{2}+s_{3}\right)\left(s_{1}+s_{2}+s_{3}\right)\right)}{2\left(s_{4}+s_{5}-1\right)^{3}\left(s_{4}+s_{5}\right)} \\
& +\frac{x_{i}}{2} \frac{\left(2 s_{3}+s_{4}+s_{5}-1\right)\left(t_{2}+t_{3}\right)}{\left(s_{4}+s_{5}-1\right)^{2}\left(s_{4}+s_{5}\right)} \\
N_{1}^{(b 2)}= & \frac{\left(s_{3}-s_{2}\right)\left(3\left(s_{1}+s_{2}+s_{3}\right) t_{1}-t_{1}+1\right)}{\left(s_{4}+s_{5}-1\right)^{2}\left(s_{4}+s_{5}\right)}-\frac{2 s_{1}\left(s_{2}-s_{3}\right)\left(1-t_{1}\right)^{2}}{\left(s_{4}+s_{5}-1\right)^{3}\left(s_{4}+s_{5}\right)} \\
& +\frac{x_{i}}{2} \frac{2\left(s_{2}-s_{3}\right)\left(1-t_{1}\right)+\left(s_{1}+s_{2}-s_{3}\right)\left(4-3 s_{4}-3 s_{5}\right)\left(t_{1}-2\right)}{\left(s_{5}+s^{2}\left(s_{4}+s_{5}\right)\right.} \\
N_{2}^{(b 1)}= & -\frac{6\left(2 s_{3}-\left(s_{4}+s_{5}\right)\left(\left(1-3 t_{1}\right) s_{1}-t_{1}\left(s_{2}-s_{3}\right)+1\right)\right)}{\left(s_{4}+s_{5}\right)^{2}} \\
& +\frac{s_{1} t_{1}\left(s_{2}-s_{3}\right)\left(20 t_{1}-3\right)}{\left(s_{4}+s_{5}-1\right)^{3}}+\frac{s_{1}\left(s_{2}-s_{3}\right)\left(6\left(1-5 t_{1}\right)\left(s_{4}+s_{5}\right)+7\right)}{\left(s_{4}+s_{5}\right)^{2}} \\
& +\frac{3\left(s_{2}-s_{3}\right)\left(t_{1}\left(s_{4}+s_{5}\right)-1\right)}{\left(s_{4}+s_{5}-1\right)^{3}\left(s_{4}+s_{5}\right)} \\
& -x_{i} \frac{2 s_{1}\left(5-3 t_{1}\right)\left(s_{4}+s_{5}\right)+s_{1}\left(1-s_{1}-2 s_{3}\right)+\left(s_{2}-s_{3}+1\right)\left(s_{2}+s_{3}\right)}{\left(s_{4}+s_{5}-1\right)^{2}\left(s_{4}+s_{5}\right)^{2}} \\
N_{2}^{(b 2)}= & -\frac{s_{1}\left(t_{2}+t_{3}\right)^{2}\left(s_{2}-s_{3}\right)}{\left(s_{4}+s_{5}-1\right)^{3}\left(s_{4}+s_{5}\right)}+\frac{2\left(s_{2}-s_{3}\right)\left(1-3 t_{1}\left(s_{4}+s_{5}\right)\right)}{\left(s_{4}+s_{5}-1\right)\left(s_{4}+s_{5}\right)^{2}} \\
& +\frac{2 s_{1}\left(s_{2}-s_{3}\right)}{\left(s_{4}+s_{5}-1\right)^{2}\left(s_{4}+s_{5}\right)^{2}} \\
& -x_{i}\left(\frac{3 t_{1}}{s_{4}+s_{5}}-\frac{3 t_{1} s_{5}\left(s_{1}+s_{2}-1\right)-3 t_{3} s_{4}+2\left(s_{1}+s_{3}-1\right)}{(\mathrm{A} .18}\right) \\
&
\end{aligned}
$$


Open Access. This article is distributed under the terms of the Creative Commons Attribution License (CC-BY 4.0), which permits any use, distribution and reproduction in any medium, provided the original author(s) and source are credited.

\section{References}

[1] ATLAS collaboration, Observation of a new particle in the search for the Standard Model Higgs boson with the ATLAS detector at the LHC, Phys. Lett. B 716 (2012) 1 [arXiv: 1207.7214] [INSPIRE].

[2] CMS collaboration, Observation of a new boson at a mass of $125 \mathrm{GeV}$ with the CMS experiment at the LHC, Phys. Lett. B 716 (2012) 30 [arXiv:1207.7235] [INSPIRE].

[3] P. Minkowski, $\mu \rightarrow$ er at a Rate of One Out of $10^{9}$ Muon Decays?, Phys. Lett. B 67 (1977) 421 [INSPIRE].

[4] T. Yanagida, Horizontal Symmetry And Masses Of Neutrinos, Conf. Proc. C 7902131 (1979) 95 [INSPIRE].

[5] M. Gell-Mann, P. Ramond and R. Slansky, Complex Spinors and Unified Theories, Conf. Proc. C 790927 (1979) 315 [arXiv:1306.4669] [inSPIRE].

[6] S.L. Glashow, The Future of Elementary Particle Physics, NATO Sci. Ser. B 61 (1980) 687 [INSPIRE].

[7] R.N. Mohapatra and G. Senjanović, Neutrino Mass and Spontaneous Parity Violation, Phys. Rev. Lett. 44 (1980) 912 [INSPIRE].

[8] J. Schechter and J.W.F. Valle, Neutrino Masses in $\mathrm{SU}(2) \times \mathrm{U}(1)$ Theories, Phys. Rev. D 22 (1980) 2227 [INSPIRE].

[9] J. Schechter and J.W.F. Valle, Neutrino Decay and Spontaneous Violation of Lepton Number, Phys. Rev. D 25 (1982) 774 [inSPIRE].

[10] T. Asaka, S. Blanchet and M. Shaposhnikov, The $\nu M S M$, dark matter and neutrino masses, Phys. Lett. B 631 (2005) 151 [hep-ph/0503065] [INSPIRE].

[11] R.N. Mohapatra and J.W.F. Valle, Neutrino Mass and Baryon Number Nonconservation in Superstring Models, Phys. Rev. D 34 (1986) 1642 [InSPIRE].

[12] M.C. Gonzalez-Garcia and J.W.F. Valle, Fast Decaying Neutrinos and Observable Flavor Violation in a New Class of Majoron Models, Phys. Lett. B 216 (1989) 360 [INSPIRE].

[13] F. Deppisch and J.W.F. Valle, Enhanced lepton flavor violation in the supersymmetric inverse seesaw model, Phys. Rev. D 72 (2005) 036001 [hep-ph/0406040] [INSPIRE].

[14] M.B. Gavela, T. Hambye, D. Hernandez and P. Hernández, Minimal Flavour Seesaw Models, JHEP 09 (2009) 038 [arXiv:0906.1461] [INSPIRE].

[15] A. Ibarra, E. Molinaro and S.T. Petcov, TeV Scale See-Saw Mechanisms of Neutrino Mass Generation, the Majorana Nature of the Heavy Singlet Neutrinos and $(\beta \beta)_{0 \nu}$-Decay, JHEP 09 (2010) 108 [arXiv: 1007.2378] [INSPIRE].

[16] A. Abada and M. Lucente, Looking for the minimal inverse seesaw realisation, Nucl. Phys. B 885 (2014) 651 [arXiv:1401.1507] [INSPIRE].

[17] S.M. Barr, A different seesaw formula for neutrino masses, Phys. Rev. Lett. 92 (2004) 101601 [hep-ph/0309152] [INSPIRE]. 
[18] M. Malinsky, J.C. Romao and J.W.F. Valle, Novel supersymmetric $\mathrm{SO}(10)$ seesaw mechanism, Phys. Rev. Lett. 95 (2005) 161801 [hep-ph/0506296] [INSPIRE].

[19] A. Abada, G. Arcadi and M. Lucente, Dark Matter in the minimal Inverse Seesaw mechanism, JCAP 10 (2014) 001 [arXiv:1406.6556] [INSPIRE].

[20] E.K. Akhmedov, V.A. Rubakov and A.Yu. Smirnov, Baryogenesis via neutrino oscillations, Phys. Rev. Lett. 81 (1998) 1359 [hep-ph/9803255] [INSPIRE].

[21] L. Canetti, M. Drewes and M. Shaposhnikov, Sterile Neutrinos as the Origin of Dark and Baryonic Matter, Phys. Rev. Lett. 110 (2013) 061801 [arXiv:1204.3902] [INSPIRE].

[22] L. Canetti, M. Drewes, T. Frossard and M. Shaposhnikov, Dark Matter, Baryogenesis and Neutrino Oscillations from Right Handed Neutrinos, Phys. Rev. D 87 (2013) 093006 [arXiv: 1208.4607] [INSPIRE].

[23] A. Abada, G. Arcadi, V. Domcke and M. Lucente, Lepton number violation as a key to low-scale leptogenesis, JCAP 11 (2015) 041 [arXiv:1507.06215] [INSPIRE].

[24] L. Canetti, M. Drewes and B. Garbrecht, Probing leptogenesis with GeV-scale sterile neutrinos at LHCb and Belle II, Phys. Rev. D 90 (2014) 125005 [arXiv:1404.7114] [INSPIRE].

[25] T.A. Mueller et al., Improved Predictions of Reactor Antineutrino Spectra, Phys. Rev. C 83 (2011) 054615 [arXiv:1101.2663] [INSPIRE].

[26] P. Huber, On the determination of anti-neutrino spectra from nuclear reactors, Phys. Rev. C 84 (2011) 024617 [Erratum ibid. C 85 (2012) 029901] [arXiv: 1106. 0687] [INSPIRE].

[27] G. Mention et al., The Reactor Antineutrino Anomaly, Phys. Rev. D 83 (2011) 073006 [arXiv:1101.2755] [INSPIRE].

[28] LSND collaboration, A. Aguilar-Arevalo et al., Evidence for neutrino oscillations from the observation of anti-neutrino(electron) appearance in a anti-neutrino(muon) beam, Phys. Rev. D 64 (2001) 112007 [hep-ex/0104049] [INSPIRE].

[29] MiniBoonE collaboration, A.A. Aguilar-Arevalo et al., A Search for electron neutrino appearance at the $\Delta m^{2} \sim 1 \mathrm{eV}^{2}$ scale, Phys. Rev. Lett. 98 (2007) 231801 [arXiv:0704.1500] [INSPIRE].

[30] MiniBoonE collaboration, A.A. Aguilar-Arevalo et al., Event Excess in the MiniBooNE Search for $\bar{\nu}_{\mu} \rightarrow \bar{\nu}_{e}$ Oscillations, Phys. Rev. Lett. 105 (2010) 181801 [arXiv:1007.1150] [INSPIRE].

[31] MiniBoonE collaboration, A.A. Aguilar-Arevalo et al., Improved Search for $\bar{\nu}_{\mu} \rightarrow \bar{\nu}_{e}$ Oscillations in the MiniBooNE Experiment, Phys. Rev. Lett. 110 (2013) 161801 [arXiv: 1207.4809] [INSPIRE].

[32] M.A. Acero, C. Giunti and M. Laveder, Limits on $\nu_{e}$ and $\bar{\nu}_{e}$ disappearance from Gallium and reactor experiments, Phys. Rev. D 78 (2008) 073009 [arXiv:0711.4222] [INSPIRE].

[33] C. Giunti and M. Laveder, Statistical Significance of the Gallium Anomaly, Phys. Rev. C 83 (2011) 065504 [arXiv: 1006.3244] [InSPIRE].

[34] A. Kusenko, Sterile neutrinos: The dark side of the light fermions, Phys. Rept. 481 (2009) 1 [arXiv:0906.2968] [INSPIRE].

[35] K.N. Abazajian et al., Light Sterile Neutrinos: A White Paper, arXiv:1204.5379 [InSPIRE]. 
[36] P.S.B. Dev, A. Pilaftsis and U.-k. Yang, New Production Mechanism for Heavy Neutrinos at the LHC, Phys. Rev. Lett. 112 (2014) 081801 [arXiv: 1308.2209] [InSPIRE].

[37] A. Das, P.S. Bhupal Dev and N. Okada, Direct bounds on electroweak scale pseudo-Dirac neutrinos from $\sqrt{s}=8 \mathrm{TeV}$ LHC data, Phys. Lett. B 735 (2014) 364 [arXiv:1405.0177] [INSPIRE].

[38] A. Das and N. Okada, Improved bounds on the heavy neutrino productions at the LHC, Phys. Rev. D 93 (2016) 033003 [arXiv:1510.04790] [InSPIRE].

[39] LBNE collaboration, C. Adams et al., The Long-Baseline Neutrino Experiment: Exploring Fundamental Symmetries of the Universe, arXiv:1307.7335 [INSPIRE].

[40] W. Bonivento et al., Proposal to Search for Heavy Neutral Leptons at the SPS, arXiv:1310.1762 [INSPIRE].

[41] A. Blondel et al., Research Proposal for an Experiment to Search for the Decay $\mu \rightarrow$ eee, arXiv:1301.6113 [INSPIRE].

[42] F.F. Deppisch, P.S. Bhupal Dev and A. Pilaftsis, Neutrinos and Collider Physics, New J. Phys. 17 (2015) 075019 [arXiv: 1502.06541] [INSPIRE].

[43] A. Atre, T. Han, S. Pascoli and B. Zhang, The Search for Heavy Majorana Neutrinos, JHEP 05 (2009) 030 [arXiv:0901.3589] [INSPIRE].

[44] S. Alekhin et al., A facility to Search for Hidden Particles at the CERN SPS: the SHiP physics case, arXiv:1504.04855 [INSPIRE].

[45] SHiP collaboration, M. Anelli et al., A facility to Search for Hidden Particles (SHiP) at the CERN SPS, arXiv:1504.04956 [inSPIRE].

[46] S. Banerjee, P.S.B. Dev, A. Ibarra, T. Mandal and M. Mitra, Prospects of Heavy Neutrino Searches at Future Lepton Colliders, Phys. Rev. D 92 (2015) 075002 [arXiv:1503.05491] [INSPIRE].

[47] A. Das and N. Okada, Inverse seesaw neutrino signatures at the LHC and ILC, Phys. Rev. D 88 (2013) 113001 [arXiv: 1207.3734] [INSPIRE].

[48] A. de Gouvêa and A. Kobach, Global Constraints on a Heavy Neutrino, Phys. Rev. D 93 (2016) 033005 [arXiv: 1511.00683] [INSPIRE].

[49] D. Ng and J.N. Ng, A Note on Majorana neutrinos, leptonic CKM and electron electric dipole moment, Mod. Phys. Lett. A 11 (1996) 211 [hep-ph/9510306] [INSPIRE].

[50] J.P. Archambault, A. Czarnecki and M. Pospelov, Electric dipole moments of leptons in the presence of Majorana neutrinos, Phys. Rev. D 70 (2004) 073006 [hep-ph/0406089] [INSPIRE].

[51] W.-F. Chang and J.N. Ng, Charged lepton electric dipole moments from TeV scale right-handed neutrinos, New J. Phys. 7 (2005) 65 [hep-ph/0411201] [INSPIRE].

[52] J.R. Ellis, J. Hisano, M. Raidal and Y. Shimizu, Lepton electric dipole moments in nondegenerate supersymmetric seesaw models, Phys. Lett. B 528 (2002) 86 [hep-ph/0111324] [INSPIRE].

[53] I. Masina, Lepton electric dipole moments from heavy states Yukawa couplings, Nucl. Phys. B 671 (2003) 432 [hep-ph/0304299] [INSPIRE].

[54] Y. Farzan and M.E. Peskin, The Contribution from neutrino Yukawa couplings to lepton electric dipole moments, Phys. Rev. D 70 (2004) 095001 [hep-ph/0405214] [INSPIRE]. 
[55] A. Ilakovac and A. Pilaftsis, Flavor violating charged lepton decays in seesaw-type models, Nucl. Phys. B 437 (1995) 491 [hep-ph/9403398] [INSPIRE].

[56] R. Alonso, M. Dhen, M.B. Gavela and T. Hambye, Muon conversion to electron in nuclei in type-I seesaw models, JHEP 01 (2013) 118 [arXiv:1209.2679] [INSPIRE].

[57] M.C. Gonzalez-Garcia, M. Maltoni and T. Schwetz, Updated fit to three neutrino mixing: status of leptonic CP-violation, JHEP 11 (2014) 052 [arXiv:1409.5439] [INSPIRE].

[58] M. Pospelov and A. Ritz, Electric dipole moments as probes of new physics, Annals Phys. 318 (2005) 119 [hep-ph/0504231] [INSPIRE].

[59] T. Fukuyama, Searching for New Physics beyond the Standard Model in Electric Dipole Moment, Int. J. Mod. Phys. A 27 (2012) 1230015 [arXiv:1201.4252] [INSPIRE].

[60] Particle Data Group collaboration, K.A. Olive et al., Review of Particle Physics, Chin. Phys. C 38 (2014) 090001 [INSPIRE].

[61] ACME collaboration, J. Baron et al., Order of Magnitude Smaller Limit on the Electric Dipole Moment of the Electron, Science 343 (2014) 269 [arXiv:1310.7534] [INSPIRE].

[62] Muon (G-2) collaboration, G.W. Bennett et al., An Improved Limit on the Muon Electric Dipole Moment, Phys. Rev. D 80 (2009) 052008 [arXiv:0811.1207] [inSPIRE].

[63] BeLLe collaboration, K. Inami et al., Search for the electric dipole moment of the tau lepton, Phys. Lett. B 551 (2003) 16 [hep-ex/0210066] [INSPIRE].

[64] O.W. Greenberg, CPT violation implies violation of Lorentz invariance, Phys. Rev. Lett. 89 (2002) 231602 [hep-ph/0201258] [INSPIRE].

[65] W.C. Griffith, Measurements and implications of of EDMs, plenary talk at Interplay between Particle 83 Astroparticle physics 2014, Queen Mary University of London, 18-22 August 2014, https://indico.ph.qmul.ac.uk/indico/conferenceDisplay.py?confId=1.

[66] J-PARC G-'2/EDM collaboration, N. Saito, A novel precision measurement of muon g-2 and EDM at J-PARC, AIP Conf. Proc. 1467 (2012) 45.

[67] E.P. Shabalin, Electric Dipole Moment of Quark in a Gauge Theory with Left-Handed Currents, Sov. J. Nucl. Phys. 28 (1978) 75 [Yad. Fiz. 28 (1978) 151] [InSPIRE].

[68] A. Pilaftsis, Resonant CP-violation induced by particle mixing in transition amplitudes, Nucl. Phys. B 504 (1997) 61 [hep-ph/9702393] [INSPIRE].

[69] W. Buchmüller and M. Plümacher, CP asymmetry in Majorana neutrino decays, Phys. Lett. B 431 (1998) 354 [hep-ph/9710460] [INSPIRE].

[70] R. Mertig, M. Böhm and A. Denner, FEYN CALC: Computer algebraic calculation of Feynman amplitudes, Comput. Phys. Commun. 64 (1991) 345 [InSPIRE].

[71] A. Abada, D. Bečirević, M. Lucente and O. Sumensari, Lepton flavor violating decays of vector quarkonia and of the $Z$ boson, Phys. Rev. D 91 (2015) 113013 [arXiv:1503.04159] [INSPIRE].

[72] MEG collaboration, J. Adam et al., New constraint on the existence of the $\mu^{+} \rightarrow e^{+} \gamma$ decay, Phys. Rev. Lett. 110 (2013) 201801 [arXiv:1303.0754] [INSPIRE].

[73] A.M. Baldini et al., MEG Upgrade Proposal, arXiv:1301.7225 [INSPIRE].

[74] SINDRUM collaboration, U. Bellgardt et al., Search for the Decay $\mu^{+} \rightarrow e^{+} e^{+} e^{-}$, Nucl. Phys. B 299 (1988) 1 [INSPIRE]. 
[75] DELPHI collaboration, P. Abreu et al., Search for neutral heavy leptons produced in $Z$ decays, Z. Phys. C 74 (1997) 57 [Erratum ibid. C 75 (1997) 580] [InSPIRE].

[76] L3 collaboration, O. Adriani et al., Search for isosinglet neutral heavy leptons in Z0 decays, Phys. Lett. B 295 (1992) 371 [INSPIRE].

[77] FCC-Ee study Team collaboration, A. Blondel et al., Search for Heavy Right Handed Neutrinos at the FCC-ee, arXiv:1411.5230 [INSPIRE].

[78] A. Abada, V. De Romeri, S. Monteil, J. Orloff and A.M. Teixeira, Indirect searches for sterile neutrinos at a high-luminosity Z-factory, JHEP 04 (2015) 051 [arXiv:1412.6322] [INSPIRE].

[79] A. Abada, A.M. Teixeira, A. Vicente and C. Weiland, Sterile neutrinos in leptonic and semileptonic decays, JHEP 02 (2014) 091 [arXiv: 1311.2830] [INSPIRE].

[80] A. Abada, D. Das, A.M. Teixeira, A. Vicente and C. Weiland, Tree-level lepton universality violation in the presence of sterile neutrinos: impact for $R_{K}$ and $R_{\pi}$, JHEP 02 (2013) 048 [arXiv: 1211.3052] [INSPIRE].

[81] T. Asaka, S. Eijima and K. Takeda, Lepton Universality in the $\nu M S M$, Phys. Lett. B 742 (2015) 303 [arXiv: 1410.0432] [INSPIRE].

[82] S. Antusch, J.P. Baumann and E. Fernandez-Martinez, Non-Standard Neutrino Interactions with Matter from Physics Beyond the Standard Model, Nucl. Phys. B 810 (2009) 369 [arXiv:0807.1003] [INSPIRE].

[83] S. Antusch and O. Fischer, Non-unitarity of the leptonic mixing matrix: Present bounds and future sensitivities, JHEP 10 (2014) 094 [arXiv:1407.6607] [INSPIRE].

[84] M.S. Chanowitz, M.A. Furman and I. Hinchliffe, Weak Interactions of Ultraheavy Fermions. 2., Nucl. Phys. B 153 (1979) 402 [InSPIRE].

[85] L. Durand, J.M. Johnson and J.L. Lopez, Perturbative Unitarity Revisited: A New Upper Bound on the Higgs Boson Mass, Phys. Rev. Lett. 64 (1990) 1215 [InSPIRE].

[86] J.G. Korner, A. Pilaftsis and K. Schilcher, Leptonic flavor changing Z0 decays in $\mathrm{SU}(2) \times \mathrm{U}(1)$ theories with right-handed neutrinos, Phys. Lett. B 300 (1993) 381 [hep-ph/9301290] [INSPIRE].

[87] J. Bernabeu, J.G. Korner, A. Pilaftsis and K. Schilcher, Universality breaking effects in leptonic $Z$ decays, Phys. Rev. Lett. 71 (1993) 2695 [hep-ph/9307295] [InSPIRE].

[88] S. Fajfer and A. Ilakovac, Lepton flavor violation in light hadron decays, Phys. Rev. D 57 (1998) 4219 [INSPIRE].

[89] A. Ilakovac, Lepton flavor violation in the standard model extended by heavy singlet Dirac neutrinos, Phys. Rev. D 62 (2000) 036010 [hep-ph/9910213] [INSPIRE].

[90] M. Blennow, E. Fernandez-Martinez, J. Lopez-Pavon and J. Menendez, Neutrinoless double beta decay in seesaw models, JHEP 07 (2010) 096 [arXiv: 1005.3240] [INSPIRE].

[91] A. Abada, V. De Romeri and A.M. Teixeira, Effect of steriles states on lepton magnetic moments and neutrinoless double beta decay, JHEP 09 (2014) 074 [arXiv: 1406.6978] [INSPIRE].

[92] GERDA collaboration, M. Agostini et al., Results on Neutrinoless Double- $\beta$ Decay of ${ }^{76} G e$ from Phase I of the GERDA Experiment, Phys. Rev. Lett. 111 (2013) 122503 [arXiv: 1307.4720] [INSPIRE]. 
[93] EXO-200 collaboration, M. Auger et al., Search for Neutrinoless Double-Beta Decay in ${ }^{136}$ Xe with EXO-200, Phys. Rev. Lett. 109 (2012) 032505 [arXiv:1205.5608] [INSPIRE].

[94] EXO-200 collaboration, J.B. Albert et al., Search for Majorana neutrinos with the first two years of EXO-200 data, Nature $\mathbf{5 1 0}$ (2014) 229 [arXiv:1402.6956] [INSPIRE].

[95] KamLAND-Zen collaboration, A. Gando et al., Limit on Neutrinoless $\beta \beta$ Decay of ${ }^{136}$ Xe from the First Phase of KamLAND-Zen and Comparison with the Positive Claim in ${ }^{76} \mathrm{Ge}$, Phys. Rev. Lett. 110 (2013) 062502 [arXiv:1211.3863] [INSPIRE].

[96] Muon G-2 collaboration, G.W. Bennett et al., Final Report of the Muon E821 Anomalous Magnetic Moment Measurement at BNL, Phys. Rev. D 73 (2006) 072003 [hep-ex/0602035] [INSPIRE]. 